\title{
High variability in Vela X-1: giant flares and off states
}

\author{
I. Kreykenbohm ${ }^{1,2,3,4}$, J. Wilms ${ }^{1,2}$, P. Kretschmar ${ }^{5}$, J. M. Torrejón ${ }^{6,7}$, K. Pottschmidt ${ }^{8,9}$, M. Hanke ${ }^{1,2}$, A. Santangelo ${ }^{3}$, \\ C. Ferrigno ${ }^{3,4,10}$, and R. Staubert ${ }^{3}$
}

1 Dr. Karl Remeis-Sternwarte Bamberg, Sternwartstrasse 7, 96049 Bamberg, Germany

2 Erlangen Centre for Astroparticle Physics (ECAP), Erwin-Rommel-Str. 1, 91058 Erlangen, Germany e-mail: Ingo.Kreykenbohm@sternwarte.uni-erlangen.de

3 Kepler Center for Astro and Particle Physics, Institut für Astronomie und Astrophysik, Sand 1, 72076 Tübingen, Germany

4 INTEGRAL Science Data Centre, 16 ch. d'Écogia, 1290 Versoix, Switzerland

5 European Space Agency, European Space Astronomy Centre, Villafranca del Castillo, PO Box 78, 28691 Villanueva de la Cañada, Madrid, Spain

${ }^{6}$ Departamento de Física, Ingeniería de Sistemas y Teoría de la Señal, Escuela Politénica Superior, Universidad de Alicante, Ap. 99, 03080 Alicante, Spain

7 Kavli Institute for Astrophysics and Space Research, Massachusetts Institute of Technology, Cambridge, MA 02139, USA

8 CRESST, University of Maryland, Baltimore County, 1000 Hilltop Circle, Baltimore, MD 21250, USA

9 NASA Goddard Space Flight Center, Astrophysics Science Division, Code 661, Greenbelt, MD 20771, USA

10 IASF - INAF, via Ugo la Malfa 153, 90136 Palermo, Italy

Received 11 April 2008 / Accepted 2 August 2008

\section{ABSTRACT}

\begin{abstract}
Aims. We investigate the spectral and temporal behavior of the high mass X-ray binary Vela X-1 during a phase of high activity, with special focus on the observed giant flares and off states.

Methods. INTEGRAL observed Vela X-1 in a long almost uninterrupted observation for two weeks in 2003 Nov/Dec. The data were analyzed with OSA 7.0 and FTOOLS 6.2. We derive the pulse period, light curves, spectra, hardness ratios, and hardness intensity diagrams, and study the eclipse.

Results. In addition to an already high activity level, Vela X-1 exhibited several intense flares, the brightest ones reaching a maximum intensity of more than $5 \mathrm{Crab}$ in the $20-40 \mathrm{keV}$ band and several off states where the source was no longer detected by INTEGRAL. We determine the pulse period to be $283.5320 \pm 0.0002 \mathrm{~s}$, which is stable throughout the entire observation. Analyzing the eclipses provided an improvement in the ephemeris. Spectral analysis of the flares indicates that there appear to be two types of flares: relatively brief flares, which can be extremely intense and show spectral softening, in contrast to high intensity states, which are longer and show no softening.

Conclusions. Both flares and off states are interpreted as being due to a strongly structured wind of the optical companion. When Vela X-1 encounters a cavity with strongly reduced density, the flux will drop triggering the onset of the propeller effect, which inhibits further accretion, giving rise to off states. The sudden decrease in the density of the material required to trigger the propeller effect in Vela X-1 is of the same order as predicted by theoretical papers about the densities in OB star winds. A similarly structured wind can produce giant flares when Vela X-1 encounters a dense blob in the wind.
\end{abstract}

Key words. X-rays: stars - stars: flare - stars: pulsars: individual: Vela X-1 - stars: magnetic fields

\section{Introduction}

Vela X-1 (4U 0900-40) is an eclipsing high mass X-ray binary (HMXB) consisting of the B0.5Ib super giant HD 77581 and a neutron star with an orbital period of 8.964 days (van Kerkwijk et al. 1995) at a distance of $\sim 2.0 \mathrm{kpc}$ (Nagase 1989). The optical companion has a mass of $\sim 23 M_{\odot}$ and a radius of $\sim 30 R_{\odot}$ (van Kerkwijk et al. 1995). Due to the small separation of the binary system with an orbital radius of just $1.7 R_{\star}$, the massive 1.9 $M_{\odot}$ neutron star (Vela X-1 is the most massive compact object known to be a neutron star; Quaintrell et al. 2003; Barziv et al. 2001) is deeply embedded in the dense stellar wind of its optical companion HD $77581\left(\dot{M}_{\star}=4 \times 10^{-6} M_{\odot} \mathrm{yr}^{-1}\right.$; Nagase et al. 1986). X-ray lines indicate that this wind is inhomogeneous with many dense clumps (Oskinova et al. 2007) embedded in a far thinner, highly ionized component (Sako et al. 1999).

The neutron star revolves with a long spin period of $\sim 283 \mathrm{~s}$ (Rappaport \& McClintock 1975; McClintock et al. 1976). Both the spin period and spin period derivative have changed erratically since their first measurements, as expected for a windaccreting system. The evolution of the spin period is most appropriately described by a random walk model (Tsunemi 1989; Ziółkowski 1985). Although the source exhibits strong pulse-topulse variations, a pulse-profile folded over several pulse periods shows remarkable stability (for 10 pulses or more; Staubert et al. 1980), even over decades (Raubenheimer 1990). At energies below $5 \mathrm{keV}$, the pulse-profile consists of a complex five-peaked structure, which transforms at energies above $20 \mathrm{keV}$ into a simple double-peaked pulse-profile (Staubert et al. 1980) where the two peaks are thought to be due to the two accreting magnetic poles of the neutron star.

With $\sim 4 \times 10^{36} \mathrm{erg} \mathrm{s}^{-1}$, the X-ray luminosity of Vela X-1 is typical of a high mass X-ray binary. Observations in the past, however, have shown that the source is variable with observed flux reductions to less than $10 \%$ of its normal value (off states; Kreykenbohm et al. 1999; Kretschmar et al. 1999; 
Inoue et al. 1984), while periods of increased activity have also been observed during which the flux increases within an hour to a multiple of the previous value, reaching peak flux levels close to 1 Crab (Kreykenbohm et al. 1999; Haberl \& White 1990; Kendziorra et al. 1989). In this respect, Vela X-1 is similar to sources such as 4U 1700-377 and 4U 1907+09, for which low luminosity states and flares have also been observed, as is rather typical for wind-accreting systems (see e.g. Fritz et al. 2006; van der Meer et al. 2005; in 't Zand et al. 1997; Haberl et al. 1989). Although Vela X-1 is a well studied object, only observations by INTEGRAL revealed that the flares in Vela X-1 can be brighter than previously anticipated (Staubert et al. 2004; Krivonos et al. 2003). While the flaring activity is thought to be due to a strongly increased accretion rate, $\dot{M}$, the origin of the $\dot{M}$ variations is unknown.

The phase averaged X-ray spectrum of Vela X-1 was usually modeled with a power law modified at higher energies by an exponential cutoff (Tanaka 1986; White et al. 1983) or with the Negative Positive EXponential (NPEX-model; Mihara 1995). The spectrum was found to be modified by strongly orbitalphase-dependent photoelectric absorption at lower energies due to the dense stellar wind and an accretion wake trailing the neutron star (Goldstein et al. 2004; Kreykenbohm et al. 1999; Feldmeier et al. 1996; Haberl \& White 1990). At $6.4 \mathrm{keV}$, an iron fluorescence line and occasionally an iron edge at $7.27 \mathrm{keV}$ (Nagase et al. 1986) were observed in the X-ray spectrum. At higher energies, cyclotron resonant scattering features (CRSFs) between 25 and $32 \mathrm{keV}$ (Makishima et al. 1992; Choi et al. 1996; Kreykenbohm et al. 2002) and at $~ 55 \mathrm{keV}$ (Kendziorra et al. 1992; Orlandini et al. 1998; Kreykenbohm et al. 1999; La Barbera et al. 2003; Attié et al. 2004) were present, although the interpretation of the $25 \mathrm{keV}$ feature is still sometimes debated (Orlandini 2006).

The remainder of this paper is structured as follows. In Sect. 2, the data and software used are described. Section 3 describes first the temporal analysis of the data, i.e. light curves, determination of the pulse period, quasi-periodic modulations, and then the analysis of the eclipse and the spectral analysis. The results are discussed in Sect. 4 and a summary is presented in Sect. 5.

\section{Data}

\subsection{Instrument and software}

The INTEGRAL observatory (Winkler et al. 2003) is in a highly eccentric orbit with a period of $71^{\mathrm{h}} 49^{\mathrm{m}}$, ideal for long uninterrupted observations with a low X-ray background. Due to the high eccentricity of the orbit the perigee passage (when INTEGRAL is inside the radiation belts) has a duration of only $\sim 8 \mathrm{~h}$, which minimizes the time when no science observations are possible due to the high radiation background.

INTEGRAL has four science instruments, which provide coverage from $3 \mathrm{keV}$ up to $10 \mathrm{MeV}$ as well as in the optical: the imager IBIS/ISGRI (Ubertini et al. 2003, $20 \mathrm{keV}$ to $800 \mathrm{keV}$ ) with moderate energy resolution and a large effective area, the spectrometer SPI (20 keV to $10 \mathrm{MeV}$; Vedrenne et al. 2003) for the analysis of nuclear lines, the X-ray monitor JEM-X (Lund et al. 2003, $3 \mathrm{keV}$ to $35 \mathrm{keV}$ ), and the optical monitor OMC (Mas-Hesse et al. 2003). All high-energy instruments are coded-mask telescopes (see e.g. In't Zand 1992, for a review of this technique). To improve the imaging quality, the satellite performs raster observations of the vicinity of an X-ray source, retaining the target in the field-of-view of ISGRI. Due

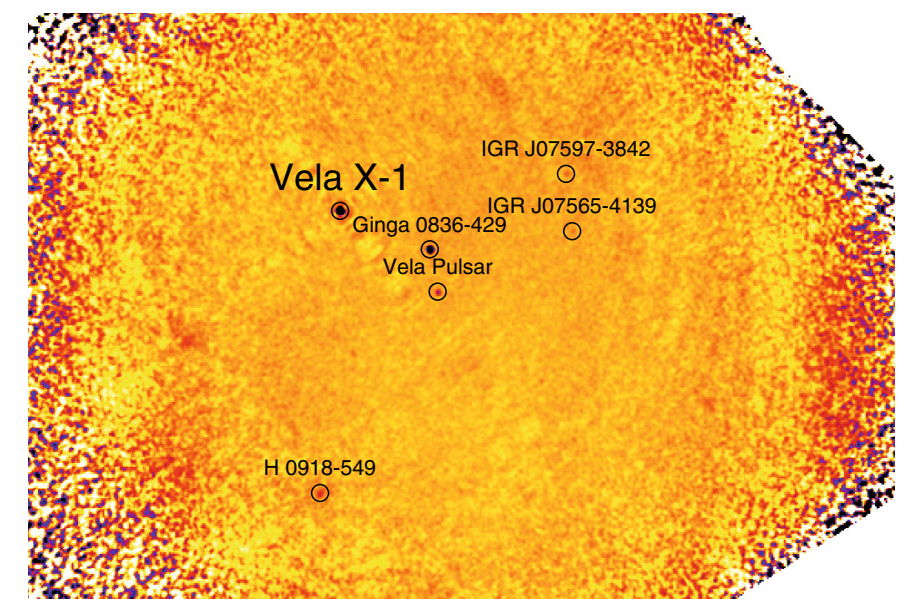

Fig. 1. Intensity mosaic image using ISGRI data of the Vela region. All five revolutions from Rev. 137 to Rev. 141 have been used for this mosaic. Vela X-1 is by far the most significant source. Several more sources are also detected. Note that the times of the eclipses of Vela X-1 (see Fig. 2) have been excluded from this mosaic, resulting in an exposure of $\sim 960 \mathrm{ksec}$. The noisy rim of the mosaic is due to the low coding factor in the outermost part of the partially coded field-of-view.

to this "dithering" strategy the off-axis angle of the target source changes significantly during the observation. When the source is more than $\sim 4.5$ away from the pointing direction, it is in the partially coded field-of-view of ISGRI. With increasing distance from the pointing direction, the coding factor decreases, causing increased uncertainties in the images, flux values, and spectra. Individual pointings made during these dithering observations are called science windows (SCWs). They have typical durations of $1800 \mathrm{~s}, 2200 \mathrm{~s}$, or $3600 \mathrm{~s}$. These SCWs are then associated with INTEGRAL revolutions, i.e. complete orbits of the INTEGRAL satellite around the Earth.

To prepare the INTEGRAL data for analysis, we used the Offline Science Analysis Software (OSA), version 7.0, and its associated calibration files. In particular, we make extensive use of the tool ii_light. We carefully checked the behavior of ii_light (see Appendix A), because the IBIS cookbook ${ }^{1}$ cautions that ii_light should only be used to analyze the timing behavior within a given science window. For further analysis, we used HEADAS release 6.2. Spectral fitting was done with XSPEC 11.3.2ad (Dorman \& Arnaud 2001; Arnaud 1996).

\subsection{Data}

As part of the AO1 core program (Winkler 2001), INTEGRAL observed the Vela region (see Fig. 1) continuously for five consecutive INTEGRAL revolutions from the beginning of revolution 137 (JD 2452970.86) until the end of revolution 141 (JD 2452970.86) resulting in approximately $1 \mathrm{Msec}$ of data (see Fig. 2). The observation was performed in a $5 \times 5$ dithering pattern with stable pointings $2^{\circ}$ apart.

We chose to use all available science windows from Rev. 137 to Rev. 141 to be able to derive a contiguous light curve with as few interruptions as possible (data gaps due to the perigee passage of the satellite are obviously unavoidable; see Fig. 2). Since Vela X-1 is a bright source, the OSA software has no problem in detecting the source and determining its flux level accurately even when the source is at an off-axis angle of more than $14^{\circ}$; in any case, fewer than $5 \%$ of the pointings had an off-axis angle

\footnotetext{
${ }_{1}$ Available at http://isdc.unige.ch/?Support+documents
} 


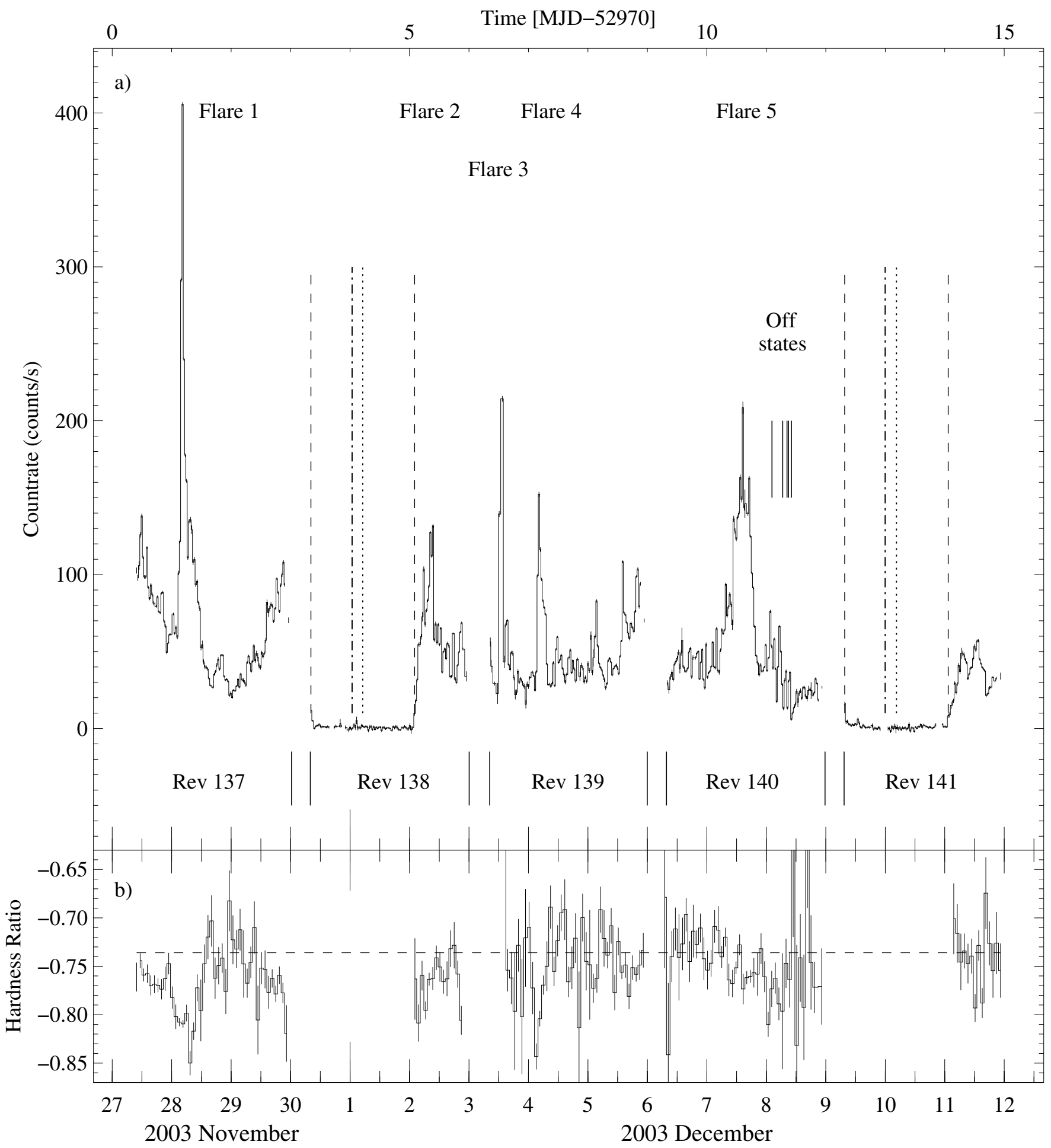

Fig. 2. Variability of Vela X-1 for the complete Vela region observation from Revolution 137 to 141 . a) ISGRI 20-40 keV light curve (time resolution $1 \mathrm{SCW}$, i.e. $\sim 1800 \mathrm{~s}$ ) and b) $20-30 \mathrm{keV}$ vs. $40-60 \mathrm{keV}$ hardness ratio as defined by Eq. (4) (rebinned by a factor of $\sim 3$ with respect to the light curve). Labels indicate the revolution number. Short vertical lines below the X-ray light curve show INTEGRAL's perigee passages, during which the instruments are switched off. The long dashed vertical lines show the ingress and egress times. The dotted vertical line indicates the derived eclipse center (see also Table 4), while the dash-dotted line indicates the time of mean longitude $T_{90}$ based on the ephemeris from Nagase (1989). Note that the offset of the newly derived $T_{90}$ (see Table 4) in comparison to that of Nagase (1989) is too small to be visible in this figure. See text for further discussion.

larger than $14^{\circ}$. For studying the timing behavior on timescales short compared with a SCW, i.e. period determination and search for QPOs (see Sect. 3.2), the absolute flux is not important and the temporal properties are unaffected by a non-optimal off-axis flux correction.
Figure 1 shows the image of the Vela region from these observations. While Vela X-1 is by far the brightest source in the field-of-view of ISGRI, we also detect 4U 0836-429 as a very prominent source reaching about a third of the average intensity of Vela X-1, and the relatively weak sources H 0918-5459, 
Table 1. Flux values of Vela X-1 in science window 013700420010 , during which giant flare 1 reached its maximum. The increase indicates the factor by which Vela X-1 was brighter during the flare than during its normal state. The flux values are averages for the entire science window. For the peak flux reached in the pulses, see Table 2.

\begin{tabular}{ccccc}
\hline \hline $\begin{array}{c}\text { Energy } \\
{[\mathrm{keV}]}\end{array}$ & $\begin{array}{c}\text { Flux (normal) } \\
{\left[10^{-10} \mathrm{erg} \mathrm{cm}^{-2} \mathrm{~s}^{-1}\right]}\end{array}$ & $\begin{array}{c}\text { Flux (flare) } \\
{[\mathrm{Crab}]}\end{array}$ & $\begin{array}{c}\text { Flux (flare) } \\
\text { Increase }\end{array}$ \\
\hline $20-30$ & 10.7 & 136.3 & 2.8 & 13 \\
$30-40$ & 5.6 & 58.6 & 1.9 & 10 \\
$40-50$ & 1.9 & 16.6 & 0.7 & 9 \\
$50-60$ & 0.6 & 4.5 & 0.3 & 8 \\
$60-80$ & 0.7 & 2.6 & 0.1 & 4 \\
$80-100$ & 0.1 & 0.6 & $<0.1$ & 6 \\
\hline
\end{tabular}

the Vela Pulsar, and two sources first reported by INTEGRAL (den Hartog et al. 2004; Sazonov et al. 2005). Since the two brightest sources Vela X-1 and 4U 0836-429 are well separated (about 6.7) and all the weaker sources are even more distant, contamination of the spectrum of Vela X-1 due to the presence of the other sources is of no concern. Data from JEM-X and SPI have not been used in this analysis due to the far smaller field-ofview of JEM-X and since Vela X-1 is off-center in the observed field (Vela X-1 was only within the fully coded field-of-view of JEM-X for less than ten out of the $\sim 550$ individual pointings). The SPI instrument, on the other hand, provides a high spectral resolution, although, due to its low effective area, it is not possible to study data on timescales of seconds as required here.

\section{Data analysis}

\subsection{Light curves and flux}

Vela X-1 was found in a strongly variable state during the Nov./Dec. 2003 observation by INTEGRAL. While periods of increased activity were observed before (Kreykenbohm et al. 1999; Haberl 1994), the behavior found in this observation (see also Staubert et al. 2004) is indeed extreme.

Most prominently, on 2003 November 28 (JD 2452971.67), INTEGRAL observed the brightest flare ever seen from Vela X-1 (designated flare 1; see Fig. 2 and also Krivonos et al. 2003). During the flare, the $20-40 \mathrm{keV}$ count rate increased from a SCW averaged pre-flare value of $\sim 55$ counts s$^{-1}(\sim 300 \mathrm{mCrab}$, or $1.6 \times 10^{-9} \mathrm{erg} \mathrm{s}^{-1}$ ) by a factor of more than seven to 405 counts s$^{-1}(2.3 \mathrm{Crab})$ within only $90 \mathrm{mn}$ - normal flaring activity reaches peak flux values not higher than $1 \mathrm{Crab}$ (see e.g. Kreykenbohm et al. 1999). Vela X-1 was therefore more than ten times brighter than on average (see Table 1) which implies that flare 1 is a giant flare. The average flux values for Vela X-1 in Table 1 were derived from a spectrum with $400 \mathrm{ksec}$ of exposure obtained between revolution 81 and 89 in June/July 2003, when Vela X-1 was in a normal state. In the following, we designate a flare with a peak flux of more than $2 \mathrm{Crab}$ as a giant flare, as opposed to normal flares, which do not reach this flux level. These flares reach their peak rapidly, i.e. $T_{\text {rise }} / T_{\text {total }}<0.3$, where $T_{\text {rise }}$ is the time from the onset of the flare to the peak. While flare 1 was detected in all energy bands, it was most pronounced in the 20-30 keV band. As stated above, Vela X-1 was never in the field-of-view of JEM-X during the flare, such that coverage at even lower energies is unavailable.

After the peak (duration about half an hour, see Fig. 3), the flare decayed quickly in less than $2 \mathrm{~h}$ to an intensity level of

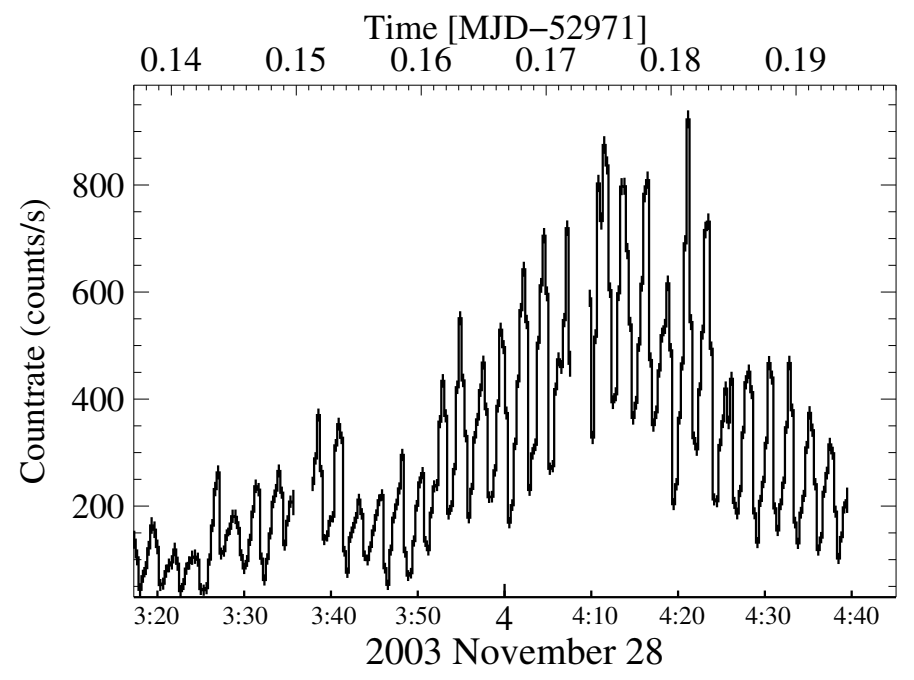

Fig. 3. Close up of the light curve with a time resolution of $20 \mathrm{~s}$ of the peak of giant flare 1 on 2003 November 28. The peak is reached at MJD 52971.18 with 923 counts s$^{-1}$ (corresponding to $5.2 \mathrm{Crab}$ ) in the 20 to $40 \mathrm{keV}$ band. This value is significantly higher than the count rate averaged over one science window, since the count rate is not constant. Furthermore, the source is strongly pulsating, exhibiting the well known double pulse.

Table 2. Overview of the observed flares. See Fig 2 for the numbering of the flares. The time is the onset of the flare. To obtain the peak fluxes $F_{\text {peak }}$, a light curve with a time resolution of $20 \mathrm{~s}$ was used. $T_{\text {rise }}$ is the time from the onset of the flare to the peak, while $T_{\text {total }}$ is the duration of the flare.

\begin{tabular}{|c|c|c|c|c|c|}
\hline Flare & $\begin{array}{l}\text { Time } \\
{[\mathrm{MJD}]}\end{array}$ & $\begin{array}{c}\text { Duration } \\
\text { [s] }\end{array}$ & $\begin{array}{c}F_{\text {peak }} \\
{[\mathrm{Crab}]}\end{array}$ & $\frac{T_{\text {rise }}}{T_{\text {total }}}$ & Remarks \\
\hline 1 & 52971.15 & 11200 & 5.2 & 0.15 & $\begin{array}{l}\text { giant flare, spectral } \\
\text { softening }\end{array}$ \\
\hline 2 & 52975.34 & 5200 & 2.6 & 0.83 & no spectral change \\
\hline 3 & 52976.50 & 1800 & 5.3 & 0.28 & giant flare, very short \\
\hline 4 & 52977.15 & 12900 & 1.9 & 0.13 & spectral softening \\
\hline 5 & 52980.31 & 31400 & 3.9 & 0.63 & $\begin{array}{l}\text { high intensity state, } \\
\text { no spectral change }\end{array}$ \\
\hline
\end{tabular}

$<1 \mathrm{Crab}$ and within $\sim 11 \mathrm{~h}$ to a SCW averaged count rate of $\sim 35$ counts s$^{-1}$ (200 mCrab), somewhat lower than before the onset of the flare (see Fig. 2). The total energy released between $20 \mathrm{keV}$ and $40 \mathrm{keV}$ amounts to $1.15 \times 10^{41} \mathrm{erg}$. We emphasize that these fluxes and those given in Fig. 2 and Table 1 are SCW averaged fluxes. These values average over fluctuations on shorter timescales such as pulsations, during which the source sometimes reached significantly higher intensities (see below and Fig. 3).

In the following days, three more flares (flares 2 to 4 , see Fig. 2), as listed in Table 2, were observed. All three flares were shorter and less intense than flare 1 on a science window averaged basis, but still reached SCW averaged intensities close to 1 Crab. Unfortunately, INTEGRAL was in engineering mode during flare 3 and the standard OSA pipeline rejects these data; therefore, only count rates could be obtained and no further analysis of this flare is possible.

On 2003 December 7 (JD 245981.10), another intense flare was observed (designated Flare 5, see Fig. 2). Unlike flare 1, during which the brightness of the source increased rapidly, it took $\sim 8 \mathrm{~h}$ for flare 5 to reach its $\mathrm{SCW}$ averaged maximum 


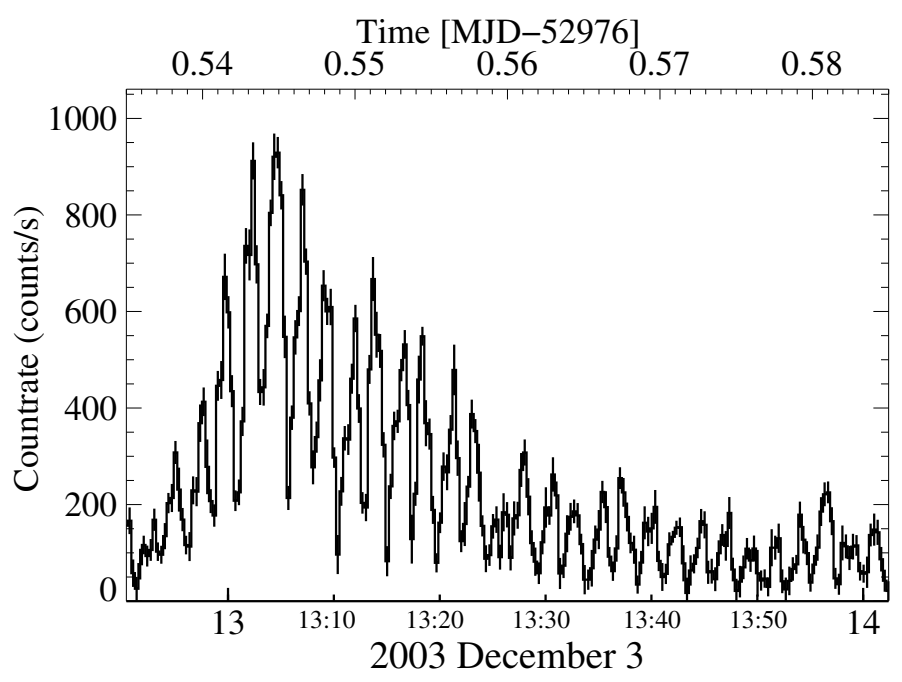

Fig. 4. ISGRI $20-40 \mathrm{keV}$ light curve of the short flare 3 on 2003 December 3 with a time resolution of $20 \mathrm{~s}$. The count rate increases from $<40$ counts s${ }^{-1}$ at the onset of the flare to 929 counts s$~^{-1}$ during the peak (thus exceeding even flare 1) in less than $800 \mathrm{~s}$ only to decay within $1200 \mathrm{~s}$ to a count rate of $<60$ counts s $\mathrm{s}^{-1}$ again. Since the flare is extremely short, its peak intensity is not evident in a light curve using SCW long bins as in Fig. 2.

20-40 keV flux of $\sim 1.2$ Crab. The decay lasted $\sim 5 \mathrm{~h}$ until Vela X-1 reached its pre-flare count rate of $\sim 35$ counts s $^{-1}$ (200 mCrab in $20-40 \mathrm{keV}$ ). Although quite bright, flare 5 is therefore significantly less intense than giant flare 1, and also far longer, i.e. it is a high intensity state. In the following, we designate flares that have a duration of many hours and exhibit a rather slow increase, i.e. $T_{\text {rise }} / T_{\text {total }} \gtrsim 0.5$, as high intensity states, as opposed to flares which exhibit a rapid increase, such as flare 1 . With $1.22 \times 10^{41} \mathrm{erg}$ the fluence of flare 5, however, was very similar to the energy release of flare 1 .

A more detailed analysis using a light curve with a $20 \mathrm{~s}$ time resolution showed that the source pulsated all the time, including during flares, and exhibited the well known strong pulse-topulse variations (Fig. 3). In the maximum, a peak $20-40 \mathrm{keV}$ count rate of $\sim 920$ counts $\mathrm{s}^{-1}$ in one $20 \mathrm{~s}$ time bin $(5.2 \mathrm{Crab})$ was observed for flare 1 and $\sim 930$ counts s$^{-1}$ (5.3 Crab) for flare 3. Flare 3 on December 3 was therefore also a giant flare (see Fig. 4). However, flare 3 was significantly shorter: the entire flare lasted less than $2000 \mathrm{~s}$, but it was as bright as flare 1 (see Table 2).

Extending the analysis to the non-flaring parts of the light curve, we detected a quasi-periodic oscillation (QPO), similar to other accreting X-ray pulsars (e.g. in 4U $0115+63$ or V 0332+53; Heindl et al. 1999; Mowlavi et al. 2006; Takeshima et al. 1994). Detecting a QPO, especially if it is transient, can be difficult. Using dynamic power spectra (PSDs), we found a short-lived QPO with a period in the range of a few $1000 \mathrm{~s}$, but no evidence for the presence of any quasi-periodic behavior below $140 \mathrm{~s}$ (half the pulse period, Sect. 3.2). The short-lived QPO with a period of $\sim 6820 \mathrm{~s}$ appears to be regular and inconsistent with pure stochastic behavior (see Fig. 5). Subsequent period searches on the corresponding data subset clearly detect the period. We note that the quasi-periodic modulation shown in Fig. 5 is far stronger and inconsistent with the NOMEX effect ${ }^{2}$, which

\footnotetext{
${ }^{2}$ For an explanation of the NOMEX support structure and its implications, see e.g. Lubiński et al. (2004) and Reglero et al. (2001).
}

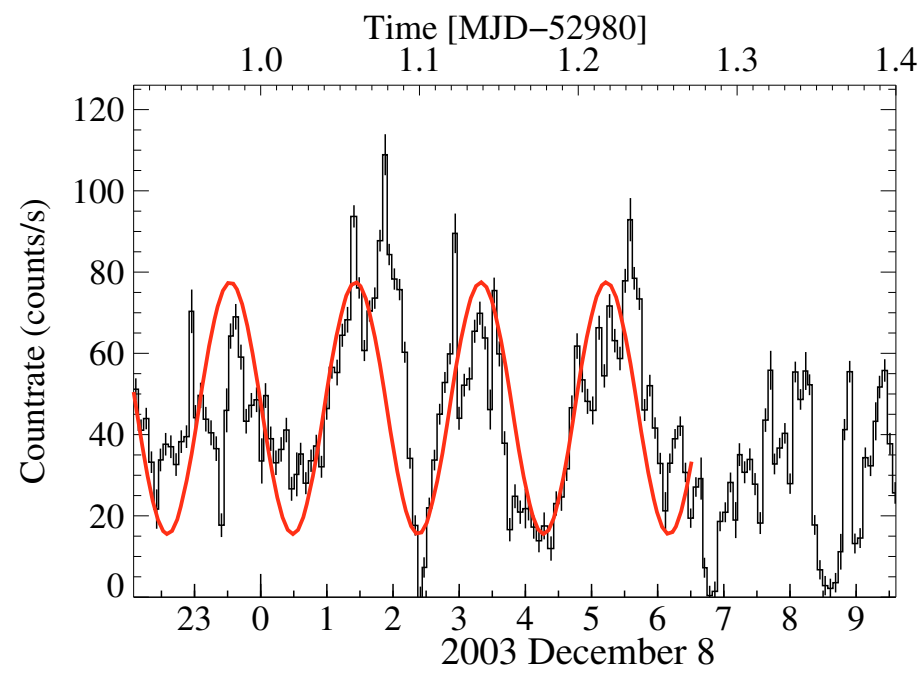

Fig. 5. A closeup of a part of the pulse averaged light curve of Vela X-1 (i.e. a light curve with a time resolution of 283 s). A quasi-periodic modulation with a period of $\sim 6820 \mathrm{~s}$ is evident in this part of the light curve, as indicated by the overplotted sine wave. Note that during the trough between $2 \mathrm{~h}$ and $3 \mathrm{~h}$, and especially following the quasi-periodic modulation, the count rate decreased several times to zero for a short time, i.e. the source became completely undetectable by ISGRI (see Table 3 and Sect. 3.1 for discussion).

can cause intensity variations from SCW to SCW, but not within a given SCW.

In this case, the amplitude of the oscillations was large, i.e. the mean count rate was $\sim 45 \mathrm{cps}$ with an amplitude of $25 \mathrm{cps}$. During the bottom part of the oscillation, however, the flux sometimes decreased to approximately zero counts for a short time (see Fig. 5), i.e. the source turns off (see the following discussion).

Although these modulations are evident (see Fig. 5), the statistics for the entire observation are weak since this event was short lived. Furthermore, it is well known that pure red noise can also produce quasi-periodic flux variations (for a discussion using Mrk 766 as an example, see Benlloch et al. 2001), therefore these modulations must be treated with care.

During and after the part of the light curve in which the quasi-periodic oscillation was present, we observed several off states, during which no significant residual flux was detectable by ISGRI and no modulation due to the otherwise omnipresent pulsations was present (Fig. 6). The onset of these off states occured without any identifiable transition phase. The source simply switches off, or more precisely, the luminosity of the source drops below the detection limit of ISGRI. The same statement is also true for the end of the off states, where Vela X-1 switches on again and immediately resumes its normal (pre off state) intensity level and behavior. We identified five such off states during the entire observation, which all occured in the $12 \mathrm{~h}$ from MJD 52981.0 and MJD 52981.5 (Table 3). Figure 6 shows off states 3 and 4, which are separated by only 600 s (about two and a half pulse periods). In between the two off states, Vela X-1 pulsated normally and at a normal intensity level. Since the two off states occured in rapid succession, they could also be considered as one longer off state interrupted by 2 normal pulses.

Off state 5 differed from the other four off states: the onset of off state 5 is also sudden, but after the pulsations are observed again, the flux level remains low for several thousand seconds. Off states 1 to 4 are shown in Fig. 5, where the flux declines to 


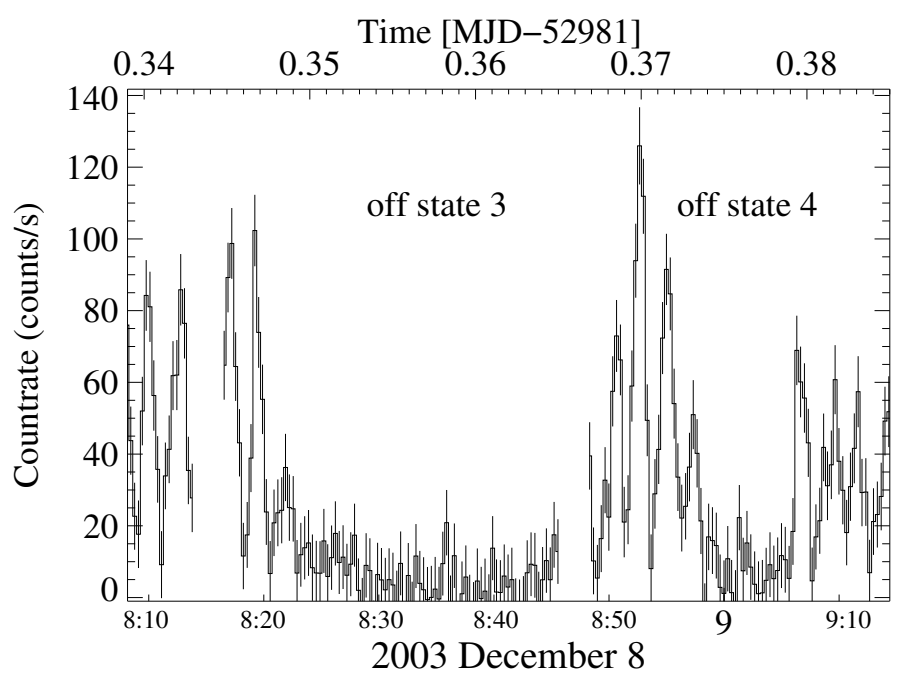

Fig. 6. 20-40 keV light curve with $20 \mathrm{~s}$ resolution of off states 3 and 4 of Vela $\mathrm{X}-1$, which are most remarkable as the source suddenly becomes undetectable by ISGRI and then turns on again two times within one hour. During the off state no pulsations are discernible, while outside the off state the double pulse-profile of Vela X-1 is visible.

Table 3. Observed off states during our Vela X-1 observation. The duration is the time between the onset of the off state (i.e. sudden intensity decrease) and end of the off state (sudden intensity increase; see Fig. 6). Off state 5 is special as the source stays at a very low luminosity level after the end of the off state; in this case, the duration equals the time when no pulsations are detected (cf. Sect. 4.3).

\begin{tabular}{cccr}
\hline \hline Off state & $\begin{array}{c}\text { Start } \\
{[\mathrm{MJD}]}\end{array}$ & $\begin{array}{c}\text { Stop } \\
{[\mathrm{MJD}]}\end{array}$ & $\begin{array}{c}\text { Duration } \\
{[\mathrm{s}]}\end{array}$ \\
\hline 1 & 52981.095 & 52981.105 & 860 \\
2 & 52981.275 & 52981.291 & 1380 \\
3 & 52981.348 & 52981.365 & 1470 \\
4 & 52981.373 & 52981.379 & 520 \\
5 & 52981.422 & 52981.445 & 1980 \\
\hline
\end{tabular}

almost zero for a short time (note, however, that the light curve in Fig. 5 has a different binning).

\subsection{Pulse period}

Due to strong pulse-to-pulse variations, the determination of the period of pulsars with long pulse periods such as Vela X-1 is rather difficult. We therefore used a two step approach. First, using epoch folding (Leahy et al. 1983), we derived an approximate period of $283.5 \pm 0.1 \mathrm{~s}$ for the Vela X-1 observation. We then used this period as a starting point for a pulseprofile template-fitting approach. In this approach we derive sets of pulse-profiles from the beginning to the end of the observation using sufficient data for the long-term pulse-profile to emerge. Since the long-term pulse-profile is known to be extremely stable (see e.g. Staubert et al. 1980; Raubenheimer 1990; Kreykenbohm et al. 2002), it was then possible to compare these pulse-profiles and determine the shift in seconds between any two profiles. Using these shifts, the starting period, and the number of elapsed pulses between the two profiles, we performed a linear fit to obtain a refined period and possibly a $\dot{P}$ with the refined period until no shift was detectable between the first profile at the start of the observation and the last profile at the end

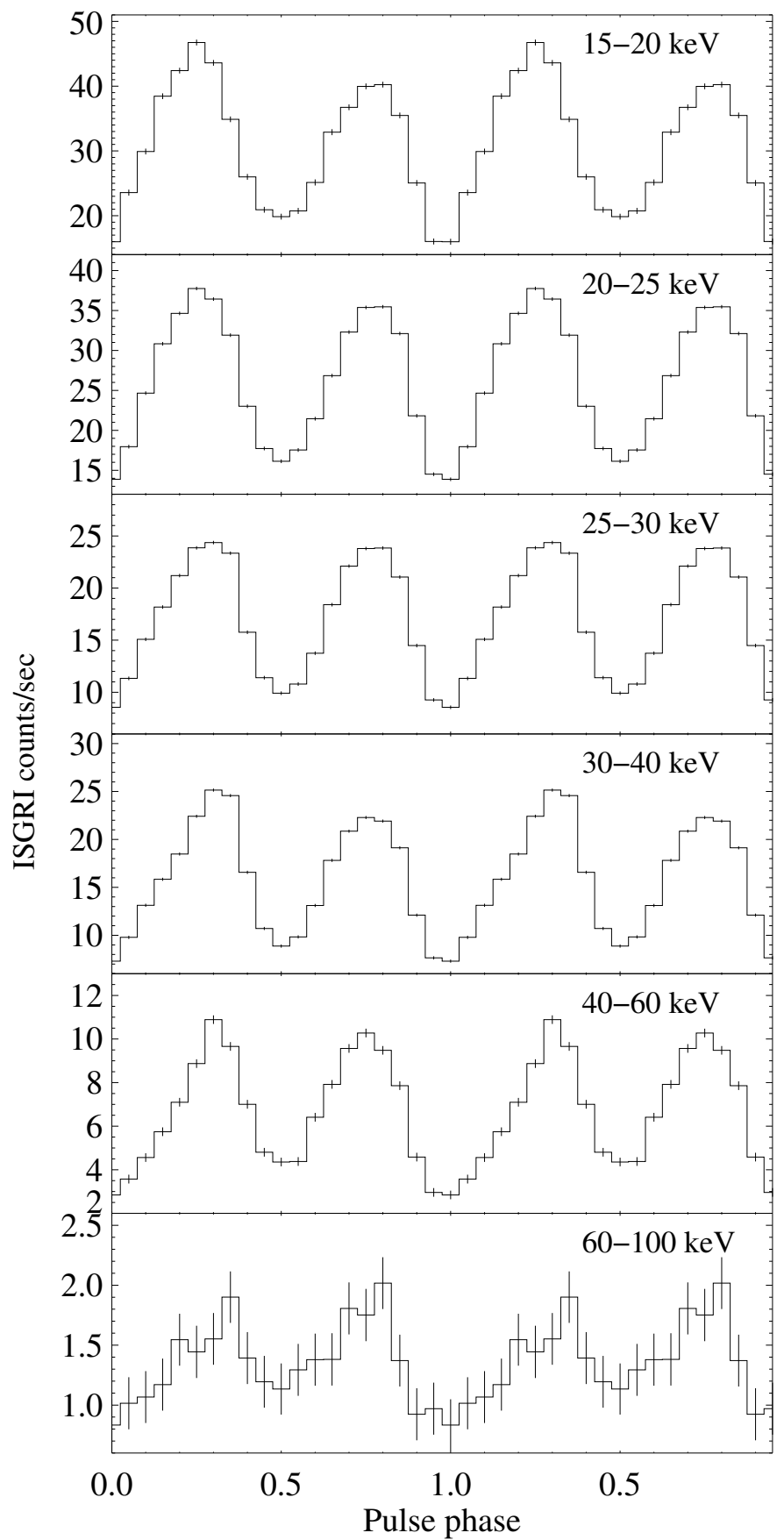

Fig. 7. Energy resolved pulse-profiles of Vela X-1 using all data outside eclipse including giant flare 1 . The folding pulse period is $283.5320 \mathrm{~s}$ (see Sect. 3.2). The profile has been adjusted such that the pulse minimum is at phase 0 . The pulses are clearly detected also in the highest energy band up to $100 \mathrm{keV}$.

of the observation (for a detailed explanation of this method, see Fritz et al. 2006). With this technique, we were able to derive a refined averaged pulse period of $283.5320 \pm 0.0002$ s. Our analysis, however, showed, that the pulse period exhibited fluctuations on timescales shorter than one orbital period with an amplitude of up to $0.003 \mathrm{~s}$ (for a detailed discussion of the evolution of the pulse period, see Staubert et al., in preparation). No pulse period "glitches" were observed, which is consistent with the short duration of the flares, during which no significant angular momentum transfer onto the neutron star is expected. 
The pulse-profile of Vela X-1 is known for its remarkable energy dependence (see for example La Barbera et al. 2003; Kreykenbohm et al. 2002, 1999; Raubenheimer 1990, and references therein) and, despite its complicated structure, also for its long-term stability (Raubenheimer 1990; Staubert et al. 1980). While the complicated profile for energies below $10 \mathrm{keV}$ consists of up to 5 peaks, the profile at energies $>20 \mathrm{keV}$ consists of two simple energy independent peaks.

The pulse-profiles shown by La Barbera et al. (2003); Kretschmar et al. (1997), and Staubert et al. (1980), however, showed that the high-energy pulse profile of Vela X-1 is also quite complex: around $30 \mathrm{keV}$, the main peak shows a triangular shape, i.e. a linear rising flank followed by a sudden and sharp fall while the secondary peak is sinusoidal (Fig. 3 of Kretschmar et al. 1997). Pulse-profiles were obtained for all data including the flares, but without the eclipses (see Fig. 7). The INTEGRAL data confirm these previous results and show that the secondary peak remains sinusoidal from $15 \mathrm{keV}$ up to $100 \mathrm{keV}$, while the main peak evolves from a simple almost sinusoidal shape around $20 \mathrm{keV}$ into a "triangular" shape with a smooth linearly rising flank followed by a steeply falling edge at $40 \mathrm{keV}$ and above. Apart from confirming earlier results, our INTEGRAL data therefore indicate that the high-energy pulse-profile is stable on timescales of decades and over significant luminosity ranges.

We also derived pulse-profiles for flare 1 only. Despite the dramatic intensity increase, however, the number of pulses added was rather small. The signal-to-noise ratio diminished, and significant systematic artefacts became evident in the folded light curve due to the low number of pulses. In any case, no significant change in the pulse-profiles was evident within the uncertainties. We therefore do not discuss these profiles further here.

\subsection{The eclipse}

During this long observation of Vela X-1, two eclipses were observed. When examining Fig. 2, the eclipses appear to be far longer than the $1.69 \pm 0.06 \mathrm{~d}$ reported by Watson \& Griffiths (1977). A detailed analysis of the "technical" circumstances, i.e. the INTEGRAL orbits and the perigee passages of the satellite, revealed that the perigee passages of INTEGRAL occur directly before both observed eclipses of Vela X-1 (see Fig. 2). Fortunately, there are a few science windows between the end of the perigee passage (i.e. the start of a new revolution) and the ingress into eclipse, such that it is possible to determine the start and end of the eclipse accurately. Since the optical companion of Vela X-1 is a massive B0.5Ib super giant with an extended variable atmosphere (Sato et al. 1986), it is difficult to determine the ingress and egress times of Vela X-1 in soft X-ray observations to high precision due to strong photoelectric absorption (see e.g. Stuhlinger et al. 2007, and references therein). At higher energies, such as in the 20-40 keV INTEGRAL data, photoelectric absorption poses less of a problem, although the determination of the egress or ingress time is only possible with an uncertainty of several $100 \mathrm{~s}$ (see Fig. 8), due to the changing extension of the atmosphere of the companion star and the general variability of the source.

Using a barycentered pulse averaged light curve, we determined the ingress and egress times of both eclipses with an accuracy of about one pulse period (see Fig. 8). Using these times, we derived the duration of the eclipses in the $20-40 \mathrm{keV}$ energy band to be $1.70 \pm 0.01 \mathrm{~d}$, consistent with the $1.69 \mathrm{~d}$ reported by Watson \& Griffiths (1977). Then we derived the center times of the two eclipses to MJD 52974.223 and MJD 52983.195, respectively. Using these two times, we derived a new mid-eclipse

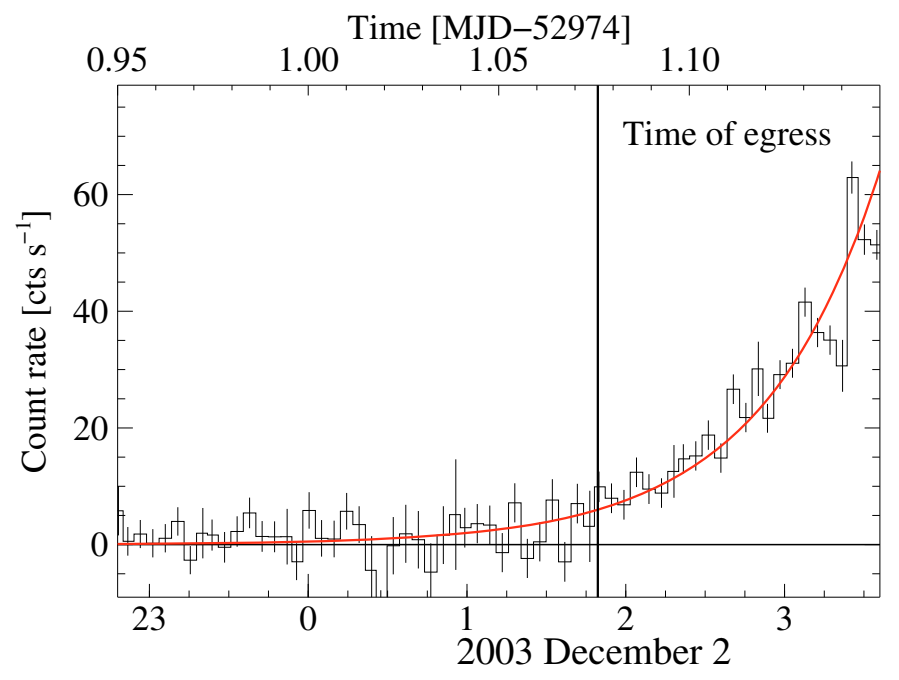

Fig. 8. Close-up of the egress of Vela X-1 from the first eclipse using a pulse averaged light curve of Vela X-1 (time resolution of $283.5 \mathrm{~s}$ ). The flux increases exponentially with time until a normal flux level is reached as indicated by the solid red line. The egress time is determined as the time when the count rate was no longer consistent with zero and is marked by a thick vertical line.

reference time of MJD 52974.227 \pm 0.007 . Note that the uncertainty in our measurement of the mid-eclipse reference time is far smaller than for earlier measurements (van der Klis \& Bonnet-Bidaud 1984; Watson \& Griffiths 1977), since the time resolution is significantly higher. To compare this mid-eclipse time with the $T_{90}$ of the ephemeris given by Bildsten et al. (1997), we need to convert the mid-eclipse time $T_{\text {ecl }}$ to the time of mean longitude $T_{90}$. Since the eccentricity $e$ of Vela X-1 is non-zero, this conversion is not straightforward. The $T_{90}$ is slightly offset from the center of the eclipse by $\Delta T=T_{\text {ecl }}-T_{90}$ (for a purely circular orbit, $T_{90}$ and $T_{\text {ecl }}$ are identical, and see also Fig. 2). This offset is given by (Deeter et al. 1987):

$\Delta T=-\frac{P_{\text {orb }}}{\pi} e \cos \omega\left(1+\frac{1}{2 \tan ^{2} i}+\frac{(\sin i-\beta)(1-\beta \sin i)}{2 \beta \sin ^{2} i}\right)$

where $\beta$ depends on the semi-major axis and the stellar radius:

$\beta=\sqrt{1-\frac{(R / a)^{2}}{1-e^{2}}}$

For the case of Vela X-1, $\beta=0.8$ (Deeter et al. 1987). Applying the corresponding values from Table 4, we obtain a significant offset of $0.226 \pm 0.005 \mathrm{~d}$ (the uncertainty is due to the uncertainty in the inclination $i$ ). We therefore calculate MJD 52974.001 \pm 0.012 to be the new measurement of $T_{90}$ (in good agreement with the $T_{90}=52974.008 \pm 0.008$ obtained from pulse-timing analysis by Staubert et al., in prep.). Comparing this $T_{90}$ with the ephemeris of Bildsten et al. (1997), we find that the time of mean longitude $T_{90}$ differs by about $0.005 \mathrm{~d}$. This shift is unsurprising given that the uncertainty in the orbital period is of the order of $0.00004 \mathrm{~d}$ and the uncertainty in $T_{90}$ of Bildsten et al. (1997) was 0.0012 d. Since then, Vela X-1 has orbited its companion 455 times such that the accumulated uncertainty amounts to a maximum shift of about $0.02 \mathrm{~d}$, far more than the observed shift of $0.005 \mathrm{~d}$. We therefore provide an updated $T_{90}$ and orbital period $P_{\text {orb }}$ for the ephemeris in Table 4, but leave the other parameters of the ephemeris unchanged. 
Table 4. Ephemeris of van Kerkwijk et al. (1995) and Bildsten et al. (1997), which have been used for obtaining the pulse period and the improved mid eclipse time $T_{\text {ecl }}$, the corresponding $T_{90}$, and the improved orbital period $P_{\text {orb}}$. See text for discussion of the new $T_{90}$.

\begin{tabular}{lcc}
\hline \hline$T_{90}$ & MJD 44278.5466 \pm 0.0037 & $(1)$ \\
$T_{90}$ & MJD 48895.2186 \pm 0.0012 & $(2)$ \\
$T_{\text {ecl }}$ & MJD 52974.227 \pm 0.007 & $(3)$ \\
$T_{90}$ & MJD 52974.001 \pm 0.012 & $(3)$ \\
$P_{\text {orb }}$ & $8.964416 \pm 0.000049 \mathrm{~d}$ & $(1)$ \\
$P_{\text {orb }}$ & $8.964368 \pm 0.000040 \mathrm{~d}$ & $(2)$ \\
$P_{\text {orb }}$ & $8.964357 \pm 0.000029 \mathrm{~d}$ & $(3)$ \\
$a \sin i$ & $113.89 \mathrm{lt}-\mathrm{sec}$ & $(2)$ \\
$i$ & $>73^{\circ}$ & $(1)$ \\
Ecc. $e$ & $0.0898 \pm 0.0012$ & $(2)$ \\
$\omega$ & $152.59 \pm 0.92$ & $(3)$ \\
\hline
\end{tabular}

References: (1) van Kerkwijk et al. (1995); (2) Bildsten et al. (1997); (3) this work.

\subsection{Spectrum}

We combined all ISGRI data outside the eclipse to create a spectrum of high signal-to-noise ratio. Since a fully working physical model of the $\mathrm{X}$-ray production mechanism in accreting $\mathrm{X}$-ray pulsars does not exist due to the complexity of the problem (significant progress has, however, been made; see e.g. Becker \& Wolff 2007), an empirical model has to be used, which consists of a power law modified by a high-energy cutoff at high energies. However, special care has to be taken not to introduce any artificial features by a "break" or a sudden onset of the cutoff as in the case of the "High-energy cutoff" in XSPEC (see for example Kreykenbohm et al. 1999, and references therein). This is true particularly if the source exhibits cyclotron resonant scattering features (CRSFs). We therefore used a power law modified by the Fermi-Dirac cutoff (Tanaka 1986) to model the spectrum. This model describes well the continuum of Vela X-1 in the ISGRI range, i.e. above $20 \mathrm{keV}$, and was in fact used successfully in the past to fit the spectrum of Vela X-1 (Kreykenbohm et al. 1999) and other sources. To model the well known CRSF in the spectrum of Vela X-1 at $50 \mathrm{keV}$ (Kendziorra et al. 1992), we used a Gaussian optical depth profile (GABS, Coburn et al. 2002). The full model for the ISGRI spectrum is then given by

$I_{\text {cont }}(E) \propto E^{-\Gamma} \times \frac{1}{\exp \left(\frac{E-E_{\text {cut }}}{E_{\text {fold }}}\right)+1} \times \exp \left(-\tau_{\mathrm{GABS}}(E)\right)$.

A systematic error of $1 \%$ was applied to account for the uncertainties in the response matrix of ISGRI.

The broad band continuum is described accurately by a power law with Fermi Dirac cutoff, but the spectral parameters were not well constrained (see Table 5). This was expected, since no data below $20 \mathrm{keV}$ were available to enable us to determine the spectral slope, the cutoff, and folding energy simultaneously, especially since the cutoff energy is expected to be around $\sim 20 \mathrm{keV}$, close to the lower end of the energy range. Fixing $\Gamma$ to some (arbitrary) value from the literature allows to $E_{\text {cut }}$ and $E_{\text {fold }}$ to be constrained more accurately, however, depending on the choice of $\Gamma$ also results in a rather bad fit (see Table 5). In any case, the parameters of the CRSF do not depend strongly on the choice of $\Gamma$.

After fitting the broadband continuum, highly significant features remain, which are due to the cyclotron line at $\sim 50 \mathrm{keV}$

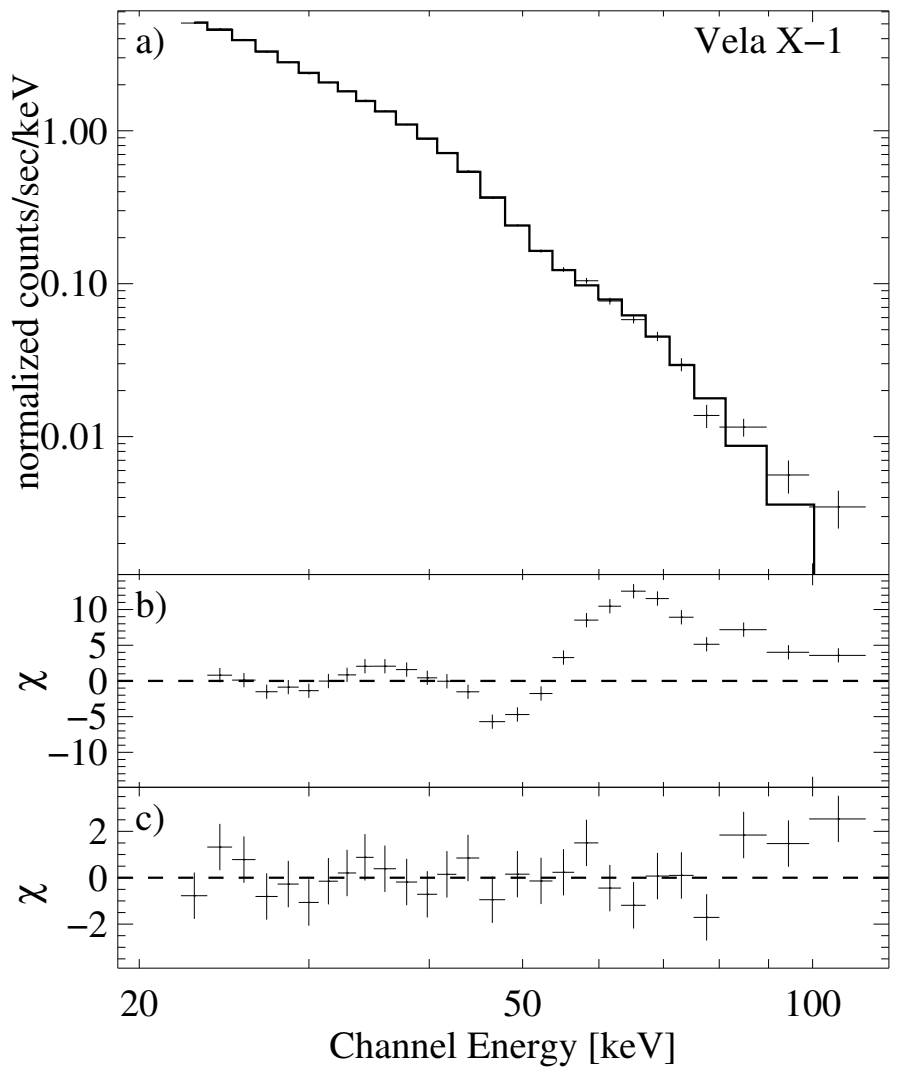

Fig. 9. Spectrum of Vela X-1 as obtained by INTEGRAL-ISGRI a) data and folded model; b) residuals when using only a power law modified by the Fermi-Dirac cutoff; c) residuals for the best-fit model with a cyclotron absorption line at $53.4_{-0.6}^{+0.7} \mathrm{keV}$. See Table 5 for all spectral parameters.

Table 5. Fit results for the overall spectrum excluding the eclipse. When fixing $\Gamma$ to one of the values found in the literature (fixed 1 and fixed 2; Kreykenbohm et al. 1999; La Barbera et al. 2003), $E_{\text {cut }}$ and $E_{\text {fold }}$ are better constrained, but the resulting $\chi_{\text {red }}^{2}$-values are higher. All uncertainties here and elsewhere in the paper are on a $90 \%$ confidence level. The d.o.f. are the degrees of freedom.

\begin{tabular}{lcrrr}
\hline \hline \multicolumn{2}{c}{ Parameter } & Free & Fixed 1 & Fixed 2 \\
Exposure & {$[\mathrm{ksec}]$} & \multicolumn{1}{c}{508} & \multicolumn{1}{c}{508} & \multicolumn{1}{c}{508} \\
\hline$\Gamma$ & & $1.6_{-0.6}^{+0.3}$ & 1.8 fix & 2.0 fix \\
$E_{\text {cut }}$ & {$[\mathrm{keV}]$} & $35.6_{-11.5}^{+7.5}$ & $41.3_{-1.5}^{+1.8}$ & $47.3_{-0.6}^{+3.5}$ \\
$E_{\text {fold }}$ & {$[\mathrm{keV}]$} & $11.2_{-0.3}^{+0.5}$ & $10.9_{-0.3}^{+0.4}$ & $10.0_{-0.6}^{+0.6}$ \\
$E_{\mathrm{C}}$ & {$[\mathrm{keV}]$} & $53.4_{-0.6}^{+0.7}$ & $53.6_{-0.6}^{+0.6}$ & $53.5_{-0.3}^{+0.9}$ \\
$\sigma_{\mathrm{C}}$ & {$[\mathrm{keV}]$} & $7.6_{-0.7}^{+0.7}$ & $8.0_{-0.5}^{+0.6}$ & $8.2_{-0.2}^{+0.9}$ \\
$\tau_{\mathrm{C}}$ & & $1.0_{-0.1}^{+0.1}$ & $1.0_{-0.1}^{+0.1}$ & $1.1_{-0.1}^{+0.2}$ \\
\hline$\chi_{\text {red }}^{2}$ (d.o.f.) & & $1.3(20)$ & $1.4(21)$ & $1.6(21)$ \\
\hline \multicolumn{2}{l}{} \\
\hline
\end{tabular}

(see Fig. 9b). After the inclusion of a Gaussian absorption line at $53.4_{-0.6}^{+0.7} \mathrm{keV}$, the resulting fit is acceptable $\left(\chi_{\text {red }}^{2}=1.3\right.$ with 20 degrees of freedom) and no significant features remain (see Fig. 9 and Table 5). Note that the shallow line like residuals below $\sim 30 \mathrm{keV}$ in Fig. $9 \mathrm{~b}$ are not due to the disputed CRSF at $25 \mathrm{keV}$ but merely a consequence of the $50 \mathrm{keV}$ line. The question of whether the $25 \mathrm{keV}$ line exists or not cannot be answered by this observation because no data below $20 \mathrm{keV}$ are available, which would be crucial to determine the continuum 
and to detect a CRSF at $25 \mathrm{keV}^{3}$. The resulting best fit is shown in Fig. 9a and the residuals in Fig 9c. Note that after the inclusion of the Gaussian absorption line at $53.4 \mathrm{keV}$, some residuals remain (see Fig. 9c) at $80 \mathrm{keV}$. In this energy range, some strong background lines are present in the ISGRI background (tungsten and lead, Terrier et al. 2003) that might be responsible for these residuals. However, it is well known that CRSFs have a complex line shape (Schönherr et al. 2007; Araya-Góchez \& Harding 2000; Araya \& Harding 1999) and are therefore not well modeled by simple Gaussian or Lorentzian functions (see for example Kreykenbohm et al. 2005; Heindl et al. 1999). The residuals present in Fig. 9c could therefore also be due to an insufficient description of the CRSF by a Gaussian absorption line and therefore incorrect modeling of the underlying continuum. To improve this unsatisfactory situation and to derive real physical parameters from the line shapes, efforts are made to create line shapes using Monte Carlo simulations (Schönherr et al. 2007).

A more detailed analysis of the spectrum using various spectral models, studying the evolution of the spectral parameters with time, and, in particular, using phase resolved spectroscopy, and a comparison with previous work is beyond the scope of this paper and will be discussed in a forthcoming publication.

\subsection{Spectral evolution during the flares}

After studying the averaged spectrum, we now consider the spectral evolution of the source during the flares, especially giant flare 1. Figure $2 b$ shows the hardness ratio over the flare, defined to be

$\mathrm{HR}=\frac{H-S}{H+S}$

where $H$ is the count rate in the hard band (40-60 keV) and $S$ the count rate in the soft band (20-30 keV). The hardness ratio remained constant throughout most of the observation, i.e. no correlation with orbital phase was evident. During the flares, the behavior of the hardness ratio, however, was remarkable. Shortly before the onset of giant flare 1 as well as during the flare, a clear deviation in the hardness ratio from its overall average value of -0.74 was evident; the hardness ratio declined suddenly to -0.82 and later during the flare even to -0.85 (see Fig. 2b). A similar behavior was observed for flare 4: with the sudden onset of the flare, the hardness ratio dropped from a pre-flare value of approximately -0.72 to -0.84 , the same level as in giant flare 1 , although flare 4 was far shorter and reached only a third of the peak flux of flare 1 . This result means that the source became significantly softer during these flares. Strikingly, however, this softening did not always occur: during flares 2 and 5, no significant change in the hardness ratio was apparent. In fact, the hardness ratio during these flares remained constant at the average value. This result is remarkable, since with a duration of $\sim 9 \mathrm{~h}$, flare 5 lasted far longer than the other flares and its peak flux reached $>50 \%$ of the peak flux of giant flare 1 ; it was therefore far longer and brighter than flare 4 , but it is associated with no spectral softening.

Although the source was bright during flares 1 and 5, it was not possible to derive meaningful spectral fits for spectra from both flares since the net exposure time was still short. We refrain from discussing the spectra of the flares in more detail since the spectral parameters could not be reliably constrained.

\footnotetext{
${ }^{3}$ Using INTEGRAL data from a 2 Msec long observation in INTEGRAL AO3 including JEM-X data (Schanne et al. 2006), however, confirmed the existence of the $25 \mathrm{keV}$ CRSF.
}

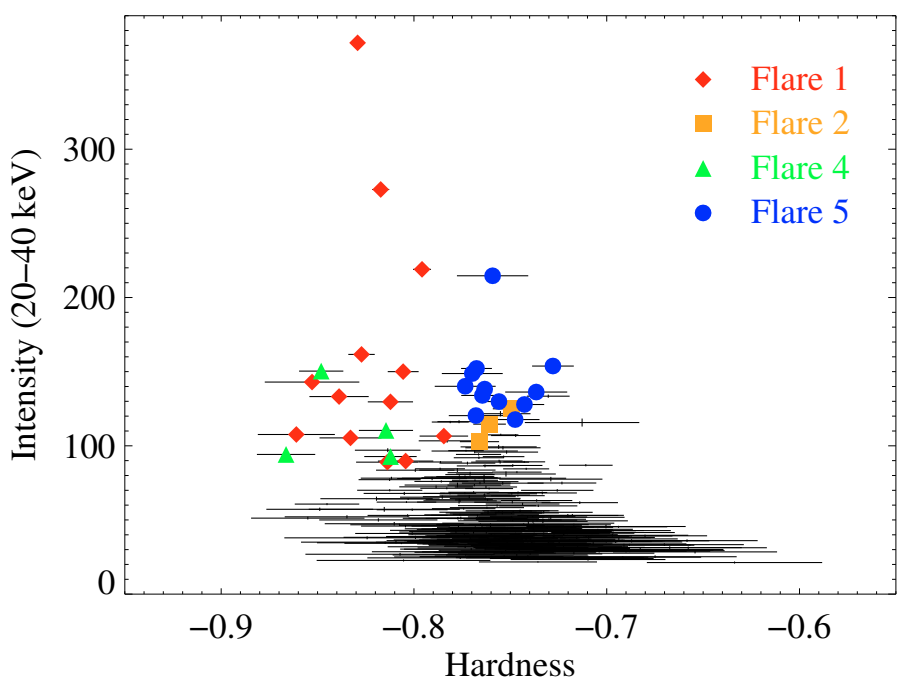

Fig. 10. Hardness Intensity Diagram of Vela X-1; data from the eclipses have been excluded. The time resolution is one science window (typically $1800 \mathrm{~s}$ ). The hardness ratio is defined as given in Eq. (4). The data points from the flares (see Fig. 2 and Table 2) are indicated by individual symbols. Note that flares 1 and 4 both show significant softening opposite to flares 2 and 5, which show no softening (see Sect. 3.5). No data are available for flare 3 (see Sect. 3.1).

"Hardness intensity diagrams" (HIDs) are a useful way to study the spectral evolution of a source. A HID for the full observation of Vela X-1, excluding the eclipses, is shown in Fig. 10. As expected, the HID of Vela X-1 does not show the $q$ shape observed for black holes (Fender \& Belloni 2004). In Vela X-1, most of the data points are clustered around the average values of intensity and hardness ratio. The only exception are the data points from giant flare 1 and the other flares, which are above the general cluster of data points due to their - by definition higher intensity. The data points of flare 1 and flare 4 are shifted, due to the spectral softening during the flare, as is already evident from the hardness ratio (see Fig. 2b). Note that the softening did not evolve during the flares, but the flares are softer than the average spectrum from the very onset. On the other hand, flares 2 and 5, although of comparable intensity, did not show any softening. Instead, the data points of the flares form two distributions (flares 1 and 4 versus flares 2 and 5) that are disjunct (see Fig. 10). On the whole, the HID shows a slight hardening towards lower count rates.

Another method of searching for spectral evolution are colorcolor diagrams (CCDs); e.g. V 0332+53 shows an interesting evolution (Reig et al. 2006). We derived a CCD for Vela X-1 but no interesting behavior was found.

\section{Discussion}

Vela X-1 has been known for a long time to have a highly timevariable light curve and to show intensity variations of up to a multiple or a fraction of the original intensity on time scales of days, hours, or even seconds.

\subsection{The flares}

Although Vela X-1 has exhibited extensive flaring activity in the past (see Kreykenbohm et al. 1999; Haberl 1994; Lapshov et al. 1992; Haberl \& White 1990; Nagase et al. 1983, among others), however, giant flares (as flares 1 and 3) had not been seen 
before. The first question is therefore whether these flares are a rare phenomenon or just could not be detected. The problem is that all these flares are rather short - even giant flare 1 and long flare 5 have a duration of a few hours. Unless the source is constantly observed as in our observation, typical sky monitors such as the All Sky Monitor (ASM) onboard RXTE fail to detect these flares. The RXTE -ASM monitors the entire observable sky regularly, typically observing Vela X-1 up to 10 times a day, but often only two or three $90 \mathrm{~s}$ long dwells per day are available ${ }^{4}$. This monitoring (which is furthermore irregular) does not allow us to detect such short-lived events as these giant flares. As shown by Staubert et al. (2004), the RXTE -ASM failed to detect the giant flares 1 and 3 when only taking $90 \mathrm{~s}$ dwells into account. During flares 2 and 5, it showed only minor increases, but given the typical uncertainty in the ASM data and the overall scatter, no unusual behavior of Vela X-1 could be detected. We note that flare 1 was present when relaxing the assumption of using only $90 \mathrm{~s}$ ASM dwells to dwells with $>80$ s exposure; however, these dwells often have larger $\chi_{\text {red }}^{2}$-values than recommended. Furthermore, detecting short-term variability with the ASM is problematic, since the ASM sometimes also erroneously detects significant flux (even at the level of the flares) while Vela X-1 is in eclipse. We therefore conclude that monitoring flares on a single dwell level with the ASM is unreliable and uninterrupted observations are required. This finding also readily explains why these giant flares have to date been unnoticed, since long uninterrupted observations spanning at least one full binary orbit of Vela X-1 are only rarely performed.

The analysis of the hardness ratio of the flares in Sect. 3.5 shows that there seem to be at least two different types of flares (see also Fig. 10): the first type (flares 1 and 4) showed dramatic increases in the count rate and the onset of the flare was very sudden. These flares showed significant spectral softening during the flare. They could appear at any time, i.e. were unrelated to the previous evolution in a similar way to flare 1 , which is superimposed on a downward trend. Although flare 4 was short, the change in the spectral hardness ratio during the flare was significant (see Fig. 2b). The second type was more similar to a high intensity state than an actual flare. Flares of this type are longer than the first type. The hardness ratio does not change during these flares, indicating that the spectrum does not change, and the source simply becomes brighter. For the short flare 3, no hardness ratio could be obtained since INTEGRAL was in engineering mode during that time; but given that it is short and features a very dramatic rise, it is likely that it belongs to the first type.

Although the hardness ratio in Fig. $2 b$ is already conclusive, a hardness ratio comparing the high-energy spectrum (above $20 \mathrm{keV}$ ) with the low-energy spectrum (below $10 \mathrm{keV}$ ) would be even more interesting since changes due to photoelectric absorption are only visible at low-energies. However, no JEM-X data are available. It is therefore impossible to determine how bright the flares were at lower energies with our current data set. Given the flux level of more than $5 \mathrm{Crab}$ in the peak of flare 1 and the significant softening observed (see Sect. 3.5), we can assume that the source was also extremely bright at lower energies. However, high photoelectric absorption could have dampened the brightness again in the classical X-ray band. The evolution of $N_{\mathrm{H}}$ during a flare could possibly help to differentiate between flare types and their underlying mechanisms, which is impossible with our current data set.

${ }^{4}$ See the RXTE-ASM project web page at http://xte.mit.edu/
The mechanism behind these different types of flares, however, is not understood. Simulating asymmetric adiabatic accretion flows onto a neutron star in the wind of OB stars, Taam \& Fryxell (1989) demonstrated that a temporary accretion disk may form in systems such as Vela X-1. The formation of this temporary accretion disk is the result of an interaction between the incident flow and shocks in the wake region and is a general property of a binary system consisting of a neutron star and an OB companion. Another consequence is a reversal of the accretion flow. Associated with the flow reversal is the destruction of the accretion disk, resulting in a significantly increased accretion rate. During this short phase, the material stored in the temporary accretion disk is accreted onto the neutron star. Taam \& Fryxell (1989) showed that these flow reversals occur on timescales of several hours, and predicted flares that would last from 15 to $60 \mathrm{mn}$, in agreement with the observation of flares 3 and 4 and also with the $1 \mathrm{~h}$ long flare observed by Kreykenbohm et al. (1999). Furthermore, the overall flaring recurrence timescale during our observation agrees with that given by Taam \& Fryxell (1989).

Later hydrodynamical studies found that wind-accretion onto the neutron star is already a highly instable process in itself. The accretion wake following the neutron star contains dense filaments of compressed gas with density variations of a factor of 100 compared with the undisturbed wind. When accreted, these density fluctuations produce abrupt changes in the X-ray luminosity (see e.g. Blondin et al. 1990). Even more important for wind-accretion, however, is the velocity structure of the wind, since

$L_{X} \propto \frac{\rho}{v^{3}}$

where $\rho$ is the density and $v$ the velocity of the wind (Bondi \& Hoyle 1944). Hydrodynamical simulations have shown that not only the density but also the velocity of the stellar wind changes dramatically with time including sharp drops and spikes (see e.g. Runacres \& Owocki 2002; Runacres \& Owocki 2005). Furthermore, the shock trailing the neutron star oscillates with brief periods of disk formation, forcing the accretion flow to change its pattern, generating the so-called "flip-flop instability" (Matsuda et al. 1991, 1987). This instability then produces disk-like rotational inflows that change their direction repeatedly (see Benensohn et al. 1997, and references therein), and is an intrinsic phenomenon that occurs whenever a gas stream flows past a neutron star or black hole, no density or velocity gradient being necessary (Matsuda et al. 1991). The timescale for this flip-flop behavior is calculated to be of the order of $45 \mathrm{~min}$ (Benensohn et al. 1997), which would agree with the flare observed by Kreykenbohm et al. (1999) and flares 3 and 4 in Fig. 2. On the other hand, the flip-flop behavior does not explain flare 1, which is superimposed on a general downward trend, because it is far longer (several hours), nor flare 5 which is about $12 \mathrm{~h}$ long, since the calculated accretion rates vary on an even shorter timescale of $\sim 100 \mathrm{~s}$ or less (see e.g. Fig. 3 of Benensohn et al. 1997).

While the above scenario explains well the short flares seen during our observation (flares 2, 3, and 4) and the overall rapid variability of Vela $\mathrm{X}-1$, the flip-flop instability fails to explain the intense long flares presented in this paper (flares 1 and 5) that last several hours; another mechanism must therefore be at work that can alter $\dot{M}$ by a factor of up to 10 , not only for $\sim 100$ s, but for many hours. Kaper et al. (1993) demonstrated that, due to the clumpiness of the shocked wind, the local density varies by a factor of 100, which can explain the flaring X-ray luminosity 
(see also Oskinova et al. 2007). Leyder et al. (2007) argued that dense clumps trapped in an otherwise thin and more homogeneous wind might be responsible for long flares, when the clumps are being accreted. Since giant flare 1 lasts several hours, however, it is unlikely that a single blob could feed the accretion for such a long time, given that the $6 \mathrm{~h}$ and $12 \mathrm{~h}$ durations of giant flare 1 and of flare 5 correspond to a considerable fraction of the neutron star orbit. Instead, the clumpy OB star wind (Kaper et al. 1993; Lucy \& White 1980) is probably viscously smeared out in a (small) accretion disk of the system. This filled accretion disk can then feed the neutron star with a significantly higher $\dot{M}$ than usual over several hours. A change of $\dot{P}$, however, cannot be observed, since the transferred angular momentum during such a flaring episode is far too small (see also Sect. 3.2). When Vela X-1 is less active, the OB star wind is probably less structured. In summary, we conclude that the observed long flares are due to a strongly structured OB star wind.

Since it is difficult to detect or monitor the flares unless during a pointed observation, it is not possible to determine how frequent such events are. Apart from the flares in our observation, reports of flares - although far less intense - are common in the literature (Kreykenbohm et al. 1999; Haberl 1994; Haberl \& White 1990, among others). In another long ( 2 Msec) observation of the Vela X-1 region in 2005 November by INTEGRAL, Vela X-1 again exhibited very intense flares (Kreykenbohm et al. 2006; Schanne et al. 2006). We therefore conclude that bright flares are quite common in Vela X-1. However, we caution that Vela X-1 does not always show high activity. During a long INTEGRAL observation in Summer 2003, Vela X-1 was in a quiet phase with only little flaring activity detected.

\subsection{Short term variability}

Although the flip-flop instability discussed in the previous section fails to explain the large flares exhibited by Vela X-1, it could explain its short-term variability: Vela X-1 exhibits strong pulse-to-pulse variations and other short-term fluctuations superimposed on the general variability, while at the same time the long-term pulse-profile remains constant over decades (Raubenheimer 1990; Kreykenbohm et al. 2002). Taam et al. (1991) developed several scenarios and the resulting behavior of the mass accretion rate. These authors obtain a time variation in $\dot{M}$ that exhibits short flare-like events on time scales of $\sim 100 \mathrm{~s}$, i.e. less than one pulse period of Vela X-1, for an accretion rate of $0.7 \dot{M}_{\mathrm{HL}}$, where $\dot{M}_{\mathrm{HL}}$ is the Hoyle-Lyttleton mass capture rate in terms of the Eddington rate (Hoyle \& Lyttleton 1941, 1939), and a wind velocity of about $1000 \mathrm{~km} \mathrm{~s}^{-1}$; such behavior is typical for O- and B-type stars and precisely that required to explain the observed short-term variability. Watanabe et al. (2006) studied the stellar wind of the Vela X-1 system and were able to explain the observed line intensities by using a wind model developed by Castor et al. (1975) with a terminal wind velocity of $1100 \mathrm{~km} \mathrm{~s}^{-1}$, which agrees with the calculations of Taam et al. (1991). Since this instability and the transfer of angular momentum appear to be intrinsic to wind-accretion (Matsuda et al. 1991), the accompanying torque reversals can therefore also be expected to exhibit randomly varying short spin-up / spin-down episodes, or a "flip-flop" behavior. As Boynton et al. (1984) suggested, this instability might therefore be identified with the random-walk behavior of the pulse period, observed in many wind-accreting sources including Vela X-1.

\subsection{The off states}

In a similar way to the flaring activity, the off states reported by Inoue et al. (1984), Kretschmar et al. (1999), and Kreykenbohm et al. (1999), where the source was not simply weaker but below the detection limit of the respective instruments and no pulsations were observed, are remarkable. After the off state observed by Kreykenbohm et al. (1999), the source resumed its normal, pulsating behavior without any transition phase (see Fig. 4 of Kreykenbohm et al. 1999); also no unusual behavior of the source was observed after the end of the off state. Inoue et al. (1984) reported that these off states occur without any prior indication. In our observation, we observed several short off states (see Table 3), which also occured without any prior indication and without a transition phase such as a slow decay. Furthermore, as shown in Fig. 5, off state 1 occurred during a phase in which the source had an average intensity level of about $250 \mathrm{mCrab}$. The count rate decreased dramatically to zero for $\sim 850 \mathrm{~s}$ (corresponding to three pulse periods) with no pulsations being visible. Kreykenbohm et al. (1999) reported observing Vela X-1 in an off state of $550 \mathrm{~s}$ (corresponding to 2 pulse periods) at the start of an observation, although the total duration of this off state is unknown, and throughout the observation, no pulsations were detected. It is possible that the events observed by Kreykenbohm et al. (1999) and in Fig. 6 are similar, i.e. the observation of the former started just in the middle of a short off state similar to that in Fig. 6.

The reasons for these off states and the sudden reappearance of pulsations are not understood, but Vela X-1 appears to experience them on a regular (but non-periodic) basis (see Fig. 6 and Table 3). Although several explanations of these phenomena have been put forth (including transiting planets, Hayakawa 1984), none can explain the observed off states, since all of these ideas (for example, variations in the mass-loss rate of the optical companion) require a significantly longer timescale. "Blobs" in the stellar wind (Feldmeier et al. 1997), for example, would not only require extremely high optical depths to block the X-rays and gamma-rays completely, but would also need very sharp borders to explain the observed sudden turn-off and turn-on of the source (sometimes within a single time bin of $20 \mathrm{~s}$, as shown in Fig. 6). We therefore consider it highly unlikely that typical clumps in the stellar wind are responsible for the off states shown in Fig. 6 and other explanations must be considered.

The wind of early-type super giants like HD 77581 is known to be inhomogeneous and clumpy (see discussion above and both Walter \& Zurita-Heras 2007; Blondin et al. 1990). Typical models show that the density in the stellar wind of super giants can vary by several orders of magnitude (Runacres \& Owocki 2005). These density variations should not only correspond to the presence of clumps - i.e. regions of strongly increased density - but also holes - regions of strongly reduced density. In these holes, the density is lower than the average density of the wind by a factor of $10^{3}$ (Runacres \& Owocki 2005). If the neutron star enters these holes, $\dot{M}$ would then also decrease by a factor of $\sim 10^{3}$ and the X-ray luminosity would be reduced accordingly. Furthermore, the density fluctuations predicted by these models occur suddenly (see Fig. 1 in Runacres \& Owocki 2005), as is the onset of the off states (see Fig. 2). On the other hand, these models predict that the density always varies; it could therefore be expected that the off states should be rather common, which does not seem to be case. Therefore, some additional mechanism must be at work that is triggered only rarely.

Another mechanism that can explain these off states is the propeller effect (Illarionov \& Sunyaev 1975). In short, the 
propeller effect inhibits the accretion of material onto the compact object when the Alfvén radius (where the ram pressure of the infalling gas and the magnetic pressure are equal) is larger than the co-rotation radius (Pringle \& Rees 1972; Lamb et al. 1973). The Alfvén radius, however, is not constant: it depends on the amount of infalling material, $\dot{M}$. If $\dot{M}$ drops, the Alfvén radius will increase and once larger than the co-rotation radius, accretion is no longer possible and the X-ray source basically switches off and no pulsations are observable. Cui (1997) observed this effect in GX 1+4: in very low luminosity states, no pulsations were observable while in high luminosity states, the source was strongly pulsating (however, other explanations are also possible to explain the absence of pulsations in GX $1+4$; see Ferrigno et al. 2007, and references therein). The magnetic field strength for which the system enters the propeller regime is then given by (Cui 1997)

$$
B=C \times\left(\frac{P}{1 \mathrm{~s}}\right)^{7 / 6} \sqrt{\frac{F_{\mathrm{X}}}{10^{-9} \mathrm{erg} \mathrm{cm}^{-2} \mathrm{~s}^{-1}}}\left(\frac{d}{1 \mathrm{kpc}}\right)\left(\frac{M}{1.4 M_{\odot}}\right)^{1 / 3}
$$

where the constant $C=4.810^{10} \mathrm{G}, P$ is the spin period of the neutron star, $F_{\mathrm{X}}$ is the bolometric X-ray flux, $d$ is the distance, and $M$ is the mass of the neutron star. Since the strength of the magnetic field of Vela X-1 is known from the observation of the cyclotron resonant scattering features (Kreykenbohm et al. 2002), Eq. (6) can be used to obtain directly the critical flux limit for the onset of the propeller effect:

$$
\begin{aligned}
F_{\mathrm{X}, \text { Propeller }}= & 4.3 \times 10^{-7} \mathrm{erg} \mathrm{cm}^{-2} \mathrm{~s}^{-1} \\
& \times\left(\frac{B}{10^{12} \mathrm{G}}\right)^{2}\left(\frac{P}{1 \mathrm{~s}}\right)^{-7 / 3}\left(\frac{d}{1 \mathrm{kpc}}\right)^{-2}\left(\frac{M}{1.4 M_{\odot}}\right)^{-2 / 3}
\end{aligned}
$$

Applying the corresponding values for Vela $X-1$, i.e. $B=2.6 \times$ $10^{12} \mathrm{G}, P=283.5 \mathrm{~s}, d=2 \mathrm{kpc}$, and $M=1.9 M_{\odot}$ (see Sect. 1 ), we obtain a critical flux of

$$
F_{\mathrm{X} \text {,Propeller,Vela X-1 }} \approx 1.1 \times 10^{-12} \mathrm{erg} \mathrm{cm}^{-2} \mathrm{~s}^{-1} \text {. }
$$

Compared with the typical bolometric flux of several times $10^{-9} \mathrm{erg} \mathrm{cm}^{-2} \mathrm{~s}^{-1}$, this critical flux is lower by about three orders of magnitude. This observed bolometric flux corresponds to an intrinsic luminosity of $\sim 6 \times 10^{32} \mathrm{erg} \mathrm{s}^{-1}$. We can therefore safely conclude that the propeller effect alone cannot force the source to switch off when Vela X-1 is in normal accretion mode.

A possible scenario would, however, be to combine both individually unsuccessful - mechanisms: the neutron star was evidently in a region in the stellar wind of strongly variable density, as demonstrated by the significant overall variability of the source and the presence of many flares. The models for the stellar wind predict density variations of up to a factor of $10^{3-5}$ (Walter \& Zurita-Heras 2007). If the neutron star enters this region with very low density, its luminosity will drop accordingly because the X-ray luminosity depends linearly on $\dot{M}$, and produce a luminosity of the order of less than $\sim 10^{33} \mathrm{erg} \mathrm{s}^{-1}$. As shown above at such low luminosity levels, Vela X-1 enters the propeller regime. This explains why no residual pulsations are observed during the off states.

Off state 5, which is considerably longer than the other off states and does not show a sudden end similar to the dip observed by Kretschmar et al. (1999), exhibits, however, a different behavior. Unlike the off states shown in Fig. 6, these dips do not show a very sudden onset or end; instead their onset (and end) is rather smooth and pulsations could also be observed for some time after the onset of the dip (see Fig. 2 in
Kretschmar et al. 1999). These authors also observed a dramatic increase in the photoelectric absorption (more than $10^{24} \mathrm{~cm}^{-2}$, Compton thick). These dips therefore belong to a second class of dips which are caused by significantly increased photoelectric absorption (Charles et al. 1978) due to an optically thick cloud in the wind of the stellar companion passing through the line-ofsight. Such clouds are known to be quite common in super-giant systems (see for example Nagase et al. 1986) and also Vela X-1 (Watanabe et al. 2006). The existence of these clouds (also referred to as blobs or clumps) in the wind of an OB stellar companion was proposed by Lucy \& White (1980), who suggested that the winds of OB stars themselves are not homogeneous, but break into a population of blobs that are radiatively driven through an ambient gas. This model was later modified to include radiatively driven shocks in the stellar wind. Since then, models of OB star winds have usually included blobs and shocks (see e.g. Feldmeier et al. 1997, and references therein). During the dip observed by Kretschmar et al. (1999), the column density, $N_{\mathrm{H}}$, was of the order of $10^{24} \mathrm{~cm}^{-2}$, similar to that measured by, for example, Leyder et al. (2007) for the density of clumps in the wind of HD 74194/IGR J08408-4503.

We therefore conclude that off states, characterized by a sudden onset and end, are usually short, and could be caused by a sudden drop in $\dot{M}$ that allows Vela X-1 to enter the propeller regime. Intensity dips, however, are significantly longer, show a smooth transition, and spectra taken during the dip provide measurements of photoelectric absorption of more than $10^{24} \mathrm{~cm}^{-2}$. These dips are readily explained by a dense blob in the wind passing through the line of sight.

\subsection{Connection with SFXTs}

The similarity between the flares and off states in Vela X-1 and the behavior of Supergiant Fast X-ray Transients (SFXTs, Sguera et al. 2005) is intriguing. SFXTs are high mass X-ray binaries that show very brief outbursts on timescales of hours or even only tens of minutes, and then remain undetectable for months between outbursts (Negueruela et al. 2008). The giant flares of Vela X-1 reported in this work are similar to these outbursts, which are assumed to be due to the accretion of a dense blob of material embedded in a thin stellar wind (Walter \& Zurita-Heras 2007): this would then imply that $L_{X}$ is a direct tracer of the density of such blobs in the stellar wind. The same holds true for Vela X-1, for which giant flares such as flare 1 are also probably due to the accretion of a dense blob in the stellar wind, as discussed above; Ferrigno et al. (2008) reached a similar conclusion in explaining the flares observed in 1E 1145.1-6141. However, we also conclude that short flares and the general variability can be explained well by the flip-flop instability, and no additional clouds in the stellar wind are necessary. Grebenev \& Sunyaev (2007) considered, why SFXTs are unobservable when they are not in outburst. According to these authors, SFXTs should be bright persistent objects since the neutron star is deeply embedded in the stellar wind. Similar to our explanation of the off states of Vela X-1, Grebenev \& Sunyaev (2007) invoke the propeller effect to explain the absence of detectable X-rays from the SFXTs when the sources are in quiescence. Unlike Vela X-1, however, SFXTs are usually in the off state, while Vela X-1 is in a normal accretion mode. The reason why SFXTs are switched off by default could either be a significantly thinner stellar wind (resulting in a lower $\dot{M}$ ) or a significantly stronger magnetic field, which would inhibit the accretion even at the densities encountered typically in the stellar wind. We therefore conclude that SFXTs and Vela X-1 are very similar 
systems, except that SFXTs are normally in the propeller regime, while Vela X-1 is normally in the accreting regime. Both, however, can switch sides, i.e. SFXTs can go into outburst, while Vela X-1 can enter the propeller regime.

\subsection{QPOs}

Several accreting X-ray pulsars show one or more quasi-periodic oscillations (QPOs) in addition to the normal pulse period (Shirakawa \& Lai 2002). Accreting X-ray pulsars that exhibit long period QPOs include 4U 0115+63 $\left(P_{\mathrm{QPO}} \sim 500 \mathrm{~s}\right.$, Heindl et al. 1999) and V 0332+53 ( $P_{\mathrm{QPO}} \sim 20 \mathrm{~s}$, Mowlavi et al. 2006; Takeshima et al. 1994) among others. The reason for the existence of QPOs is not always clear, although the $0.05 \mathrm{~Hz}$ QPO in $\mathrm{V} 0332+53$ is thought to be due to inhomogeneities in the accretion disk (Mowlavi et al. 2006), while a second QPO at $0.22 \mathrm{~Hz}$ (Qu et al. 2005) is almost (but not entirely) coincident with the pulse period of the system. It can therefore be assumed to originate in the X-ray production region, and maybe also be linked to the strong pulse-to-pulse variations in that system. In 4U 1907+09, another wind-accreting system, a transient QPO was observed with a period of $\sim 18$ s (in't Zand et al. 1998). Due to the close similarity of this system with Vela X-1, we could expect to observe a QPO with a period between $10 \mathrm{~s}$ and $40 \mathrm{~s}$. As discussed above, no evidence for a QPO with a period below $140 \mathrm{~s}$ was found. However, a QPO with a long periodicity in the range of several thousand seconds was observed instead.

The quasi-periodic behavior observed in Vela X-1 (see Fig. 5) is intriguing, albeit quite short lived. Such a periodicity - if real - in the luminosity of the source would indicate that the accretion rate onto the neutron star also varies periodically, which implies the presence of a quasi-periodic structure in the stellar wind of the companion star, either in density or in velocity or both. Such periodic structures in the wind are certainly not due to the flip-flop instability discussed above, because the instabilities produce a chaotic non-periodic modulation in the accretion rate (see e.g. Figs. 4 and 6 of Taam et al. 1991). These periodic structures could be driven by instabilities in the atmospheres of very luminous stars, which are likely to reach distances of a few stellar radii. Various mechanisms for the production of these instabilities are discussed in the literature, among them radiationpressure-driven hydrodynamical instabilities (Shaviv 2001a,b), rotational instabilities (Fullerton et al. 1997), non-radial pulsations (Owocki \& Cranmer 2002), and surface magnetic fields (Ud-Doula 2002).

One of the phenomena predicted by some models (e.g. Ud-Doula \& Owocki 2002) are "ray" like structures in the stellar wind due to the magnetic field of the OB star. It can be imagined that when the neutron star passes through these more or less periodic ray structures in the wind, the varying mass accretion rate $\dot{M}$ could then lead to the quasi-periodic behavior shown in Fig. 5. When the neutron star enters a region where the stellar wind has a significantly lower density, the accretion rate $\dot{M}$ could drop to a level at which Vela X-1 enters the propeller regime (see Sect. 4.3), as occured in off state 1 (see Fig. 5). This scenario is interesting because it helps to understand the other off states in the following part of the light curve (see Fig. 5), and to explain the density variations discussed in Sect. 4.3.

The quasi-periodic modulations in the source intensity could therefore easily be identified with similar structures in the wind. It should then be possible to draw conclusions from the period and observed intensity variations, about the applicable models and parameters and the underlying physics. However, these models, as currently present in the literature, are general and applicable numbers cannot readily be ascertained. In principle, however, as soon as these numbers can be obtained, the systematic and long-term probing of the stellar wind structure by the neutron star in Vela X-1 could provide physical constraints of the various wind models.

\section{Summary}

We have presented the analysis of a long, continuous INTEGRAL observation of Vela X-1 spanning about 1.7 binary orbits. In detail, our results are:

- Vela X-1 was found to be in a highly active state.

- The observation of several flares were recorded, two of them giant flares with a peak brightness of more than 5 Crab.

- A transient QPO with a period of $\sim 6820$ s was observed, which we attributed to inhomogeneities in the stellar wind.

- Several off states were identified during which the source becomes undetectable by INTEGRAL and no pulsations were visible. The onset of the off states was very sudden, in addition to their end.

- The pulse period was determined to be $P=283.5320 \pm$ $0.0004 \mathrm{~s}$ with no evidence for a spin-up or spin-down during the entire observation.

- Non-sinusoidal high-energy pulse-profiles were obtained up to $100 \mathrm{keV}$.

- The spectrum exhibited the well known CRSF at $53.4 \mathrm{keV}$, but due to a lack of low-energy data, the elusive line at $\sim 25 \mathrm{keV}$ could not be observed.

- Vela X-1 exhibited two types of flares: rapidly rising flares, which were correlated with a spectral change, and high intensity states, which were longer and during which the spectrum remained unchanged.

- The short flares can be explained well by the flip-flop instability: the predicted timescales of 15 to $60 \mathrm{mn}$ are in perfect agreement with the observed duration of the short flares.

- Two different types of off states exist, which are probably either caused by a dense blob blocking the line-of-sight, or the onset of the propeller effect due to a drop in $\dot{M}$.

- The similarity between Vela X-1 and SFXTs is striking: giant flares (in SFXTs: outbursts) are probably caused by the accretion of a dense blob from the stellar wind, while off states (in SFXTs: quiescence) are likely to be caused by the onset of the propeller effect.

Acknowledgements. We acknowledge financial support from DLR grants 50OG9601 and 50OG0501, NASA grant NNG05GK55G, and a travel grant from the Deutscher Akademischer Austauschdienst. I.K. acknowledges the hospitality of the Universities of Alicante, Warwick, Erlangen-Nürnberg, and the University of California at San Diego. We thank the members of the pulsar team supported by the International Space Science Institute (ISSI) in Berne, Switzerland, for discussions which greatly helped shape the ideas presented in this paper, and ISSI itself for its hospitality. J.M.T. acknowledges the support of the Spanish Ministerio de Educación y Ciencia (MEC) under grant PR2007-0176. This work is based on observations with INTEGRAL, an ESA project with instruments and science data centre funded by ESA member states (especially the PI countries: Denmark, France, Germany, Italy, Switzerland, Spain), Czech Republic and Poland, and with the participation of Russia and the USA.

\section{Appendix A: Performance of ii_light}

For our analysis of the temporal properties of Vela X-1, we require a bin size of $20 \mathrm{~s}$. The standard IBIS analysis pipeline, however, can only create light curves with a minimum bin time of at least $60 \mathrm{~s}$ and typically a bin time of $100 \mathrm{~s}$ is used. We therefore used the tool ii_light (part of OSA 7.0) to derive light 


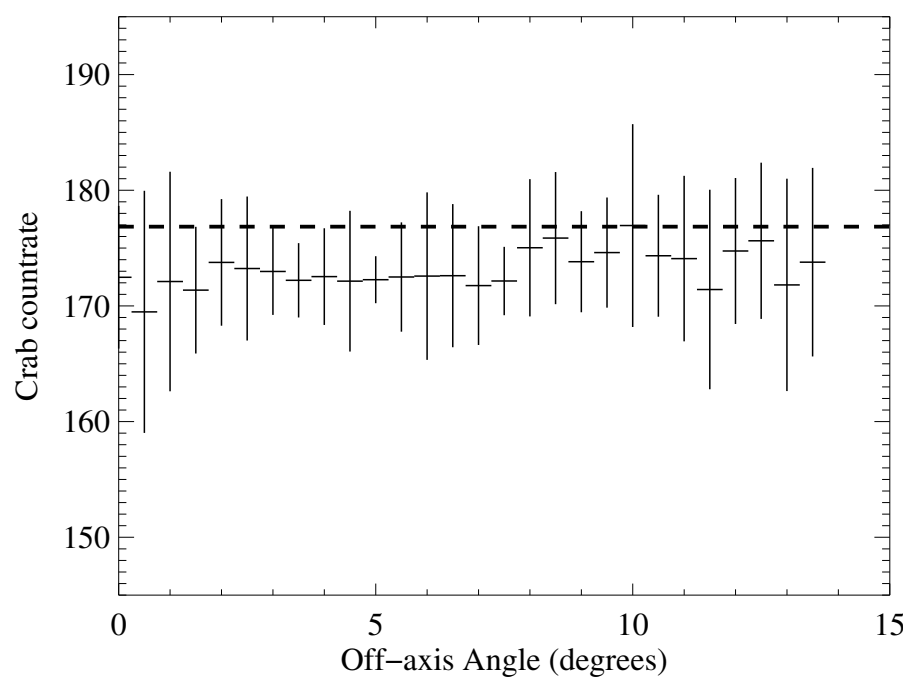

Fig. A.1. Dependence of the Crab count rate as measured by ii_light on the off-axis angle. The average count rate of the Crab when the source is in the fully coded field-of-view (4.5) as obtained by the standard IBIS pipeline is shown by the dashed line. Note that count rates obtained by ii_light are within 5\% of this nominal value even up to high offaxis angles. ii_light, however, almost always slightly underestimates the count rate compared to the standard pipeline.

curves, since it is not only efficient and convenient to use but has the capability to derive light curves with a time resolution as high as $0.1 \mathrm{~s}$. Since the IBIS cookbook cautions that ii_light should only be used to analyze the temporal behavior within a given science window, we carefully verified the performance of ii_light by using all publicly available Crab data up to revolution 464, i.e. about 1500 science windows, corresponding to an exposure time of about 3 Msec. First, we operated the standard IBIS pipeline up to the imaging level to derive background and off-axis correction maps to correct for the absorption due to the NOMEX effect (for explanation, see the IBIS analysis manual, and for the implications on the calibration, see Lubiński et al. 2004); no jumps in the count rate with changing off-axis angle were therefore observed. We then used ii_light to obtain light curves. Using the same time resolution of $20 \mathrm{~s}$ as for the Vela X-1 analysis, we derived an average count rate for the Crab of 172.5 counts s ${ }^{-1}$ between $20 \mathrm{keV}$ and $40 \mathrm{keV}$ when the source was in the fully coded field-of-view (max. off-axis angle of 4.5), which is very close to the 176.8 counts s ${ }^{-1}$ obtained by the ibis pipeline for an entire science window. Furthermore, we found that the deviation in the count rates obtained with ii_light is within five percent of the nominal value up to off-axis angles of more than $14^{\circ}$, which is of the order of the expected uncertainty (see Fig. A.1). In any case, ii_light systematically underestimates the count rate (see Fig. A.1), and the derived flux values can be considered lower limits. In summary, we found that $i i_{-}$light produces usable count rates up to an off-axis angle of $14^{\circ}$, we therefore conclude that the usage of the ii_light tool for our purposes is well justified.

\section{References}

Araya, R. A., \& Harding, A. K. 1999, ApJ, 517, 334

Araya-Góchez, R. A., \& Harding, A. K. 2000, ApJ, 544, 1067

Arnaud, K. A. 1996, in Astronomical Data Analysis Software and Systems V, ed. J. H. Jacoby, \& J. Barnes (San Francisco), ASP Conf. Ser., 101, 17

Attié, D., Chernyakova, M., Kretschmar, P., et al. 2004, in 35th COSPAR Scientific Assembly, Paris, 2862

Barziv, O., Kaper, L., Van Kerkwijk, M. H., Telting, J. H., \& Van Paradijs, J. 2001, A\&A, 377, 925
Becker, P. A., \& Wolff, M. T. 2007, ApJ, 654, 435

Benensohn, J. S., Lamb, D. Q., \& Taam, R. E. 1997, ApJ, 478, 723

Benlloch, S., Wilms, J., Edelson, R., Yaqoob, T., \& Staubert, R. 2001, ApJ, 562, 121

Bildsten, L., Chakrabarty, D., Chiu, J., et al. 1997, ApJS, 113, 367

Blondin, J. M., Kallman, T. R., Fryxell, B. A., \& Taam, R. E. 1990, ApJ, 356, 591

Bondi, H., \& Hoyle, F. 1944, MNRAS, 104, 273

Boynton, P. E., Deeter, J. E., Lamb, F. K., et al. 1984, ApJ, 283, L53

Castor, J. I., Abbott, D. C., \& Klein, R. I. 1975, ApJ, 195, 157

Charles, P. A., Watson, K. O., White, N. E., et al. 1978, MNRAS, 183, 813

Choi, C. S., Dotani, T., Day, C. S. R., \& Nagase, F. 1996, ApJ, 471, 447

Coburn, W., Heindl, W. A., Rothschild, R. E., et al. 2002, ApJ, 580, 394

Cui, W. 1997, ApJ, 482, L163

Deeter, J. E., Boynton, P. E., Lamb, F. K., \& Zylstra, G. 1987, ApJ, 314, 634 den Hartog, P. R., Hermsen, W., Kuiper, L. M., et al. 2004, ATEL, 261

Dorman, B., \& Arnaud, K. A. 2001, in Astronomical Data Analysis Software and Systems X, ed. F. R. Harnden, Jr., F. A. Primini, \& H. E. Payne, ASP Conf. Ser., 238, 415

Feldmeier, A., Anzer, U., Boerner, G., \& Nagase, F. 1996, A\&A, 311, 793 Feldmeier, A., Puls, J., \& Pauldrach, A. W. A. 1997, A\&A, 322, 878 Fender, R., \& Belloni, T. 2004, ARA\&A, 42, 317

Ferrigno, C., Segreto, A., Santangelo, A., et al. 2007, A\&A, 462, 995

Ferrigno, C., Segreto, A., Mineo, T., Santangelo, A., \& Staubert, R. 2008, A\&A, 479,533

Fritz, S., Kreykenbohm, I., Wilms, J., et al. 2006, A\&A, 458, 885

Fullerton, A. W., Massa, D. L., Prinja, R. K., Owocki, S. P., \& Cranmer, S. R. 1997, A\&A, 327, 699

Goldstein, G., Huenemoerder, D. P., \& Blank, D. 2004, AJ, 127, 2310

Grebenev, S. A., \& Sunyaev, R. A. 2007, Ast. Lett., 33, 149

Haberl, F. 1994, A\&A, 288, 791

Haberl, F., \& White, N. E. 1990, ApJ, 361, 225

Haberl, F., White, N. E., \& Kallman, T. R. 1989, ApJ, 343, 409

Hayakawa, S. 1984, Adv. Space Res., 3, 35

Heindl, W. A., Coburn, W., Gruber, D. E., et al. 1999, ApJ, 521, L49

Hoyle, F., \& Lyttleton, R. A. 1939, in Proc. Cambridge Philos. Soc., 405

Hoyle, F., \& Lyttleton, R. A. 1941, MNRAS, 101, 227

Illarionov, A. F., \& Sunyaev, R. A. 1975, A\&A, 39, 185

Inoue, H., Ogawara, Y., Ohashi, T., \& Waki, I. 1984, PASJ, 36, 709

In't Zand. 1992, Ph.D. Thesis, University of Utrecht

in 't Zand, J. J. M., Strohmayer, T. E., \& Baykal, A. 1997, ApJ, 479, L47

in't Zand, J. J. M., Baykal, A., \& Strohmeyer, T. E. 1998, ApJ, 496, 386

Kaper, L., Hammerschlag-Hensberge, G., \& van Loon, J. T. 1993, A\&A, 279, 485

Kendziorra, E., Mony, B., Maisack, M., et al. 1989, in Proc. 23rd ESLAB Symp. on Two-Topics in X-Ray Astronomy, Bologna, ESA SP-296, 467

Kendziorra, E., Mony, B., Kretschmar, P., et al. 1992, in Frontiers of X-Ray Astronomy, Proc. XXVIII Yamada Conf., ed. Y. Tanaka, \& K. Koyama, Frontiers Science Series, 2, 51

Kretschmar, P., Pan, H. C., Kendziorra, E., et al. 1997, A\&A, 325, 623

Kretschmar, P., Kreykenbohm, I., Wilms, J., et al. 1999, in Proc. 5th Compton Symposium, ed. M. McConnell, \& J. Ryan, AIP Conf. Proc., 510, 163

Kreykenbohm, I., Kretschmar, P., Wilms, J., et al. 1999, A\&A, 341, 141

Kreykenbohm, I., Coburn, W., Wilms, J., et al. 2002, A\&A, 395, 129

Kreykenbohm, I., Mowlavi, N., Produit, N., et al. 2005, A\&A, 433, L45

Kreykenbohm, I., Staubert, R., von Kienlin, A., et al. 2006, ATEL, 711

Krivonos, R., Produit, N., Kreykenbohm, I., et al. 2003, ATEL, 211

La Barbera, A., Santangelo, A., Orlandini, M., \& Segreto, A. 2003, A\&A, 400, 993

Lamb, F. K., Pethick, C. J., \& Pines, D. 1973, ApJ, 184, 271

Lapshov, I. Y., Syunyaev, R. A., Chichkov, M. A., et al. 1992, SvA Let., 18, 16

Leahy, D. A., Elsner, R. F., \& Weisskopf, M. C. 1983, ApJ, 272, 256

Leyder, J.-C., Walter, R., Lazos, M., Masetti, N., \& Produit, N. 2007, A\&A, 465, L35

Lubiński, P., Dubath, P., Kretschmar, P., Pottschmidt, K., \& Walker, R. 2004, in The INTEGRAL Universe, ed. V. Schönfelder, G. Lichti, \& C. Winkler, ESA SP-552, 871

Lucy, L. B., \& White, R. L. 1980, ApJ, 241, 300

Lund, N., Budtz-Jørgensen, C., Westergaard, N. J., et al. 2003, A\&A, 411, L231

Makishima, K., Mihara, T., Nagase, F., \& Murakami, T. 1992, in Frontiers of X-Ray Astronomy, Proc, XXVIII Yamada Conf., ed. Y. Tanaka, \& K. Koyama, Frontiers Science Series, 2, 23

Mas-Hesse, J. M., Giménez, A., Culhane, J. L., et al. 2003, A\&A, 411, L261

Matsuda, T., Inoue, M., \& Sawada, K. 1987, MNRAS, 226, 785

Matsuda, T., Sekino, N., Sawada, K., et al. 1991, A\&A, 248, 301

McClintock, J. E., Rappaport, S., Joss, P. C., et al. 1976, ApJ, 206, L99

Mihara, T. 1995, Ph.D. Thesis, RIKEN, Tokyo

Mowlavi, N., Kreykenbohm, I., Shaw, S. E., et al. 2006, A\&A, 451, 187 
Nagase, F. 1989, PASJ, 41, 1

Nagase, F., Hayakawa, S., Makino, F., Sato, N., \& Makishima, K. 1983, PASJ, 35,47

Nagase, F., Hayakawa, S., Sato, N., Masai, K., \& Inoue, H. 1986, PASJ, 38, 547

Negueruela, I., Torrejón, J. M., Reig, P., Ribó, M., \& Smith, D. M. 2008, in A population explosion: the nature and evolution of $\mathrm{X}$-ray binaries in diverse environments, ed. R. M. Bandyopadhyay, St. Petersburg, Florida, AIP Conf. Proc., 1010, 252

Orlandini, M. 2006, in Spectra \& Timing of Compact X-ray Binaries, Mumbai, India, AdvSR, 38, 2742

Orlandini, M., Dal Fiume, D., Frontera, F., et al. 1998, A\&A, 332, 121

Oskinova, L. M., Hamann, W.-R., \& Feldmeier, A. 2007, in Clumping in Hot Star Winds, ed. W.-R. Hamann, A. Feldmeier, \& L. Oskinova, Potsdam, 203

Owocki, S. P. \& Cranmer, S. R. 2002, in Radial and Nonradial Pulsations as Probes of Stellar Physics, ed. C. Aerts, T. R. Bedding, \& J. ChristensenDalsgaard, IAU Coll., 185, 512

Pringle, J. E., \& Rees, M. J. 1972, A\&A, 21, 1

Qu, J. L., Zhang, S., Song, L. M., \& Falanga, M. 2005, ApJ, 629, L33

Quaintrell, H., Norton, A. J., Ash, T. D. C., et al. 2003, A\&A, 401, 313

Rappaport, S., \& McClintock, J. E. 1975, IAU Circ., 2794

Raubenheimer, B. C. 1990, A\&A, 234, 172

Reglero, V., Sánchez, F., Rodrigo, J., et al. 2001, in Exploring the Gamma-Ray Universe, ed. A. Gimenez, V. Reglero, \& C. Winkler, Alicante, ESA SP-459, 619

Reig, P., Martínez-Núñez, S., \& Reglero, V. 2006, A\&A, 449, 703

Runacres, M. C., \& Owocki, S. P. 2002, A\&A, 381, 1015

Runacres, M. C., \& Owocki, S. P. 2005, A\&A, 429, 323

Sako, M., Liedahl, D. A., Kahn, S. M., \& Paerels, F. 1999, ApJ, 525, 921

Sato, N., Hayakawa, S., Nagase, F., et al. 1986, PASJ, 38, 731

Sazonov, S., Churazov, E., Revnivtsev, M., Vikhlinin, A., \& Sunyaev, R. 2005, A\&A, 444, L37

Schanne, S., Gotz, D., Gerard, L., et al. 2006, in The obscured Universe, ed. S. Grebenev, R. Sunyaev, \& C. Winkler, Moscow, ESA SP-622
Schönherr, G., Wilms, J., Kretschmar, P., et al. 2007, A\&A, 472, 353

Sguera, V., Barlow, E. J., Bird, A. J., et al. 2005, A\&A, 444, 221

Shaviv, N. J. 2001a, ApJ, 549, 1093

Shaviv, N. J. 2001b, MNRAS, 326, 126

Shirakawa, A., \& Lai, D. 2002, ApJ, 565, 1134

Staubert, R., Kendziorra, E., Pietsch, W. C., Reppin, J. T., \& Voges, W. 1980, ApJ, 239, 1010

Staubert, R., Kreykenbohm, I., Kretschmar, P., et al. 2004, in The INTEGRAL Universe, ed. Battrick, Munich, ESA SP-552, 259

Stuhlinger, M., Kretschmar, P., Pollock, A., et al. 2007, in The Extreme Universe in the Suzaku Era, CD-ROM, Kyoto

Taam, R. E., \& Fryxell, B. A. 1989, ApJ, 339, 297

Taam, R. E., Fu, A., \& Fryxell, B. A. 1991, ApJ, 371, 696

Takeshima, T., Dotani, T., Mitsuda, K., \& Nagase, F. 1994, ApJ, 436, 871

Tanaka, Y. 1986, in Radiation Hydrodynamics in Stars and Compact Objects, ed. D. Mihalas, \& K.-H. A. Winkler, Heidelberg, IAU Coll., 89, 198

Terrier, R., Lebrun, F., Bazzano, A., et al. 2003, A\&A, 411, L167

Tsunemi, H. 1989, PASJ, 42, 453

Ubertini, P., Lebrun, F., Di Cocco, G., et al. 2003, A\&A, 411, L131

Ud-Doula, A. 2002, Ph.D. Thesis, University of Delaware

Ud-Doula, A., \& Owocki, S. P. 2002, ApJ, 576, 413

van der Klis, M., \& Bonnet-Bidaud, J. M. 1984, A\&A, 135, 155

van der Meer, A., Kaper, L., di Salvo, T., et al. 2005, A\&A, 432, 999

van Kerkwijk, M. H., van Paradijs, J., Zuiderwijk, E. J., et al. 1995, A\&A, 303, 483

Vedrenne, G., Roques, J.-P., Schönfelder, V., et al. 2003, A\&A, 411, L63

Walter, R., \& Zurita-Heras, J. 2007, A\&A, 476, 335

Watanabe, S., Sako, M., Ishida, M., et al. 2006, ApJ, 651, 421

Watson, M. G., \& Griffiths, R. E. 1977, MNRAS, 178, 513

White, N. E., Swank, J. H., \& Holt, S. S. 1983, ApJ, 270, 711

Winkler, C. 2001, in Exploring the Gamma-Ray Universe, ed. A. Gimenez, V. Reglero, \& C. Winkler, Alicante, ESA SP-459, 471

Winkler, C., Courvoisier, T. J.-L., Di Cocco, G., et al. 2003, A\&A, 411, L1

Ziółkowski, J. 1985, Acta Astron., 35, 185 